\title{
Zu einer politischen Theorie der Marktökonomie. Theoriebildung in Zeiten der Postdemokratie
}

\author{
Frank Nullmeier
}

\section{Towards a Political Theory of the Market Economy. Theory Construction in Post-Democratic Times}

\begin{abstract}
Some authors make the dominance of markets responsible for the current crisis of Western democracies. In order to prevent a further development in a post-democratic direction political science has to scrutinize all strategies that aim to establish a predominance of politics including strategies beyond a better market regulation and a further expansion of the welfare state. The paper examines selected contributions to normative political theory in search for models of (1) the democratization of the market economy, (2) the creation of a just economy and (3) the moralization of market economies. Are justifiable models for a democratized economy, a just economy or a moralized market presented in the literature? The distinction between market organizations (corporations) and market transactions proves to be important in order to answer the question of how a reconfiguration of the market economy dominated by standards of justice and political equality could look like. Models of internal democratization and self-management have been developed for companies as the organizational part of market economies. In contrast, there is no way to democratize market transactions themselves. With reference to John Rawls and his conception of a property-owning democracy the article analyses the inherent tendency of market transactions to contribute to an accumulation of inequality and the institutional models to limit or to compensate for these side effects of markets.
\end{abstract}

Keywords: Political Theory, Rawls, Market Economy, Political Economy, Post-Democracy

Schlagwörter: Politische Theorie, Rawls, Marktwirtschaft, Politische Ökonomie, Postdemokratie

\section{Einleitung}

Im Europa der Eurozone scheint sich derzeit der Übergang zu Postdemokratien im Sinne Colin Crouchs (2008) anzubahnen. Nationalstaaten verlieren ihre Handlungsmöglichkeiten und werden auf eine Politik permanenter Austerität (Schäfer u. Streeck 2013) und Fortführung eines wirtschaftsliberalen Kurses festgelegt - trotz dessen Beitrag zur Krisenverursachung (Crouch 2011). Der Verlust an nationalstaatlicher Steuerungsfähigkeit, steigende soziale Ungleichheiten und ein fortgesetzter Prozess des Wohlfahrtsstaatsabbaus (Obinger 2012) lassen nicht erwarten, dass sich diese Untergrabung der Demokratie in Richtung postdemokratischer Zustände bald umkehren ließe. Unklar ist aber, ob sich diese Entwicklungen allein aus der Konstruktion der europäischen Währungsunion oder eher der Weltfinanzmarktkrise im Jahre 2008 (u. a. James 2009; Posner 2009) und der 
folgenden „Great Recession“ (Bermeo u. Pontusson 2012) ergeben oder gar generelle Begleiterscheinungen eines Kapitalismus darstellen, der sich immer stärker über seine in der Nachkriegszeit institutionalisierten Begrenzungen hinwegsetzt und nun auch die Einbettung in demokratische politische Systeme sprengt. Gibt es eine inhärente Tendenz eines jeden marktwirtschaftlich-kapitalistischen Systems, jede sich bietende Möglichkeit zur Expansion auf Kosten anderer institutioneller Strukturen zu nutzen, oder wirken aktuell nur situationsgebundene, eher der europäischen Teil-Integration oder dem aktuellen Typ der Finanzmarktregulierung geschuldete Entwicklungen? Je nachdem, wie die Antwort auf diese Frage ausfällt, kann man die Analyse normativer Fragen postdemokratischer Entwicklung entweder auf dem Felde der Europapolitik führen - mit Wolfgang Streeck und Fritz Scharpf als Exponenten einer Rückbesinnung auf nationalstaatliche Handlungsfähigkeit und einen Rückbau der Währungsunion (Streeck 2013; Scharpf 2013). Oder aber man lässt die Kritik des derzeitigen Finanzmarktkapitalismus in Reformkonzepten eines regulatorisch und keynesianisch ausgerichteten, weltweiten Umbaus der marktwirtschaftlichen Ökonomien münden (z. B. Stieglitz 2012). Eine dritte Option aber - und dieser Weg soll hier eingeschlagen werden - besteht darin, nach den intellektuellen Ressourcen zu fragen, die einer kritischen Bewertung von Marktwirtschaft und Kapitalismus sowie der Entwicklung von Alternativen generell zur Verfügung stehen, ganz unabhängig von den aktuellen Anlässen von Eurozonenkrise, Finanzkrise, Rezession und den aktuellen Regulationsdefiziten der Finanzmärkte. Dabei beschränkt sich dieser Beitrag darauf, innerhalb der politischen Theorie und politischen Philosophie nach Ansätzen zu suchen, die einer Kritik ökonomischer Ordnungen zuarbeiten: Verfügen wir über Argumentationspotenziale, die einem schleichenden Übergang von Demokratien zu Postdemokratien ein theoretisches Modell entgegensetzen könnten, das die Marktdynamik normativen Maßstäben, wie sie in Demokratie-, Gerechtigkeits- und Moraltheorien üblich sind, unterwerfen könnte? ${ }^{1}$ Bisher liegt der Schwerpunkt der Postdemokratie-Debatte auf der Zeitdiagnose, die Entwicklung von alternativen Entwürfen und theoretischen sowie praktischen Vorgehensweisen zur Gegenwehr fehlt weitgehend. Es bedarf daher Überlegungen zur Neufassung des Demokratieprojekts, die auch die Verursacherseite, die Marktökonomie, einbezieht. Ein Gegenentwurf zur Postdemokratie kann heute nicht mehr als politisches Projekt allein verstanden werden. Das Nachdenken über Wege zur Sicherung der politischen Demokratie verlangt, dass sich die politische Theorie mit ihren Mitteln auch auf die Ökonomie erstreckt, dass sie um Entwürfe zur normativen Bindung oder Neustrukturierung der Marktökonomie ringt. Ohne eine entwickelte politische Theorie der Restrukturierung der Marktökonomie wird sich keine normative

1 Der Beitrag verdankt sehr viel den Diskussionen im Frankfurter DFG-Forscherkolleg „Justitia Amplificata“, dessen Fellow ich im Wintersemester 2011/12 als Gast am Forschungskolleg Humanwissenschaften in Bad Homburg sein durfte. Besonderer Dank gilt den Direktoren des Forscherkollegs Stefan Gosepath und Rainer Forst, den Mitkollegiaten und auch den Frankfurter und Bad Homburger Team-Mitarbeitern und Mitarbeiterinnen sowie Thomas Biebricher und Frieder Vogelmann als Veranstaltern des Workshops „Normative Theorie und Gesellschaftskritik“ am 8. und 9.12. 2011 in Frankfurt am Main. Für eine kritische Durchsicht und Kontrolle des Manuskripts danke ich vor allem Martin Nonhoff sowie den beiden anonymen GutachterInnen. 
Konzeption mehr entwickeln lassen, die verständlich machen könnte, wie die Demokratie ihrer Aushöhlung durch Marktdynamiken entgehen sollte. $\mathrm{Zu}$ diesem Thema soll im Folgenden eine Sichtung einiger kategorialer Möglichkeiten vorgenommen werden.

Die Suche nach einer politischen Theorie der Marktökonomie muss ihren Ausgang bei der alltäglichen Trennung von Demokratie und Marktwirtschaft bzw. Kapitalismus nehmen. ${ }^{2}$ Dies entspricht der für die westlichen Gesellschaften (zu nationalen Unterschieden vgl. Münnich 2012) üblichen Denkweise, von zwei Sphären oder Funktionssystemen auszugehen, der Politik und der Ökonomie, eine Vorstellung, der auf legitimatorischer Ebene ein ,Trennmodell' entspricht: Politik (Demokratie) und Ökonomie (Marktwirtschaft) folgen jeweils eigenen Legitimitätskonzeptionen. Demokratische Gleichheit gilt als Leitwert des politischen Funktionssystems, Allokationseffizienz als Leitwert des ökonomischen Systems. Diese Konstellation wird heute sehr virtuos von Seiten der Systemtheorie verteidigt: Aus der Eigenlogik und operativen Autonomie der gesellschaftlichen Funktionssysteme mit jeweils eigenen Reflexionstheorien könne auf die funktionale Notwendigkeit systemspezifischer Legitimitätskonzepte geschlossen werden (Teubner 2012). Solange die Funktionssysteme ihre Leistungen den anderen Systemen zur Verfügung stellten, sei ein nicht konfliktfreies, aber gesamtgesellschaftlich tragfähiges Zusammenwirken möglich. Zulässig erscheint eine gewisse Ausdehnung des Funktionssystems Politik zum Ausgleich von Defiziten des Marktsystems: das gilt als Aufgabe des Sozial- und Interventionsstaates. Die expansive Tendenz der Politik dürfe aber nie so weit gehen, dass Operationsweisen des ökonomischen Systems außer Kraft gesetzt oder die Legitimitätsstandards von einem auf das andere Feld übertragen werden. Jeder Versuch, den Markt selbst sozial und demokratisch zu gestalten, aber auch jeder Versuch, die Politik wie einen Markt zu veranstalten, gelten im Modell funktionaler Differenzierung als unangemessen und potenziell destruktiv. Gegen eine derartige generalisierende Rechtfertigung der Sphärentrennung und ihrer Legitimation lässt sich eine skeptischere, weil historisch einordnende Sichtweise anführen, die davon ausgeht, dass es nur den besonders günstigen Umständen politischer und wirtschaftlicher Art nach dem Zweiten Weltkrieg zuzurechnen war, dass eine relative Einheit von Demokratie, Sozialstaat und Kapitalismus entstehen konnte. In Zeiten weniger förderlicher Randbedingungen sei dagegen damit zu rechnen, dass sich eine grundlegende Tendenz der Marktwirtschaft zur Limitation der Demokratie (so Dahl 1998, S. 178-179) oder zur Unterminierung demokratischer und sozialstaatlicher Verhältnisse entfalten werde (Crouch 2008; Streeck 2013). Hält man diese zunächst demokratieförderliche, bei weiterer Expansionstendenz einer marktwirtschaftlichen Ökonomie aber demokratiebegrenzende oder gar demokratiebedrohende Entwicklung für möglich, ist unabhängig von begünstigenden und weniger förderlichen historischen Rahmenbedingungen theoretisch zu reflektieren, ob die legitimatorische Autonomie eines gesellschaftlichen Funktionssystems Ökonomie als kapitalistische oder marktwirtschaftliche Ökonomie dauerhaft hingenommen werden kann. Im Folgenden

2 Diese beiden Begriffe werden hier zunächst als Synonyme verwendet, bei der Diskussion von Alternativen wird das Konzept sozialistischer Marktwirtschaft eingeführt. 
wird daher geprüft, welche Möglichkeiten für eine normative politische Theorie gegeben sind, die über Konzepte einer sozialstaatlichen Eingrenzung der Marktwirtschaft hinausweisen will. Allerdings steht nicht ein Überblick über das Feld der radikaleren ökonomischen Reformmaßnahmen ${ }^{3}$ und der dazugehörenden theoretischen Konzeptionen ${ }^{4}$ im Zentrum dieses Beitrags, sondern die Identifizierung von grundlegenden Theoriebildungsoptionen anhand einiger besonders einschlägiger Argumente ausgewählter Autoren.

Normative Ansätze, die die legitimatorische Autonomie der Marktökonomie in Frage stellen wollen, können auf drei theoretische Argumentationslinien zurückgreifen. Sie können sich erstens auf Demokratietheorien berufen und daraus Strategien der Demokratisierung der Ökonomie herleiten. Sie können sich zweitens auf Gerechtigkeitstheorien beziehen und nach der Gestaltung einer gerechten Ökonomie fragen. Sie können drittens auf ethische Theorien referieren, um nach Möglichkeiten einer Ethisierung oder Moralisierung der Ökonomie zu suchen. ${ }^{5}$ Diese drei Optionen prüft dieser Beitrag in den Kapiteln 2 (Demokratisierung), den Kapiteln 3 und 4 (Gerechte Ökonomie) und Kapitel 5 (Moralisierung). ${ }^{6}$ Bestandteil dieser Kapitel ist jeweils auch die Erörterung von besonders aktuellen Theoriebeiträgen, die die Begriffe Demokratie, Gerechtigkeit und Ethik in Anspruch nehmen, aber zugunsten einer Kompatibilität zum Marktgeschehen in ih-

3 Siehe vor allem: Wright 2010 oder in der deutschsprachigen Publizistik die Beiträge zur Wirtschaftsdemokratie, zur Entwicklung linker wirtschaftspolitischer Positionen und zur Bankenreform: z. B. Demirovic 2007, Bispinck et al. 2008, Martens 2010, Misik 2010, Hickel 2012, Bofinger 2012, Stiglitz 2012. Wirtschaftsdemokratische Ansätze werden in Thomas Meyers Theorie der sozialen Demokratie (2011, S. 311-312, 519) von der Warte sozialstaatlicher Versuche der Zivilisierung der kapitalistischen Ökonomie aus kritisch betrachtet. Eine kultursoziologische und zugleich wirtschaftstheoretische Kritik, wie sie Joseph Vogl liefert, zeichnet den „Einbruch unbeherrschbarer Kontingenz" (Vogl 2010, S. 171), Ungewissheit, wilder Überraschungen und schicksalhafter Zufälligkeit als Grundlage der Marktkritik auf, während Arnoldi in Anwendung der Theorie der Weltrisikogesellschaft Ulrich Becks der Finanzkrise noch positive Perspektiven in Richtung einer transnationalen Risikogemeinschaft abgewinnen kann (Arnoldi 2009, S. 86): Beide Ansätze gesellschaftstheoretischer Vergewisserung bleiben ohne Hinweis auf alternative Vergesellschaftungsformen.

4 So werden jene Beiträge, die die Frage globaler (sozialer und ökonomischer) Gerechtigkeit erörtern, in diesem Rahmen nicht systematisch einbezogen, also z. B. Arbeiten von Thomas Pogge (2008; Pogge u. Moellendorf 2008) und zu seiner Theorie (Jaggar 2010) sowie von Amartya Sen (summierend in 2009), aber auch Brock 2009 oder Miller 2010, um das dort zusätzlich auftretende Thema der Steuerungskapazitäten jenseits des Nationalstaates vermeiden zu können. Auch andere wichtige Diskussionslinien wie die weltweit aktive Bewegung zugunsten von Gemeingütern (Helfrich u. Heinrich-Böll-Stiftung 2012) in der Tradition der Theorie Elinor Ostroms bleiben hier außer Acht.

5 Die Verwendung des Begriffs Moral weicht hier von einem Sprachgebrauch ab, der Gerechtigkeit der Moraltheorie zuweist und moralische von ethischen Fragen abgrenzt. Unter dem Begriff Moralisierung sollen gerade nicht Anforderungen institutioneller Gerechtigkeit, sondern Anwendungsmöglichkeiten von Tugendethiken und personenbezogenen normativen Anforderungen verhandelt werden. Daher könnte auch von Ethisierung gesprochen werden, es wird aber der meist vertrautere Begriff der Moralisierung vorgezogen.

6 Allerdings würde nur die Möglichkeit einer Demokratisierung der Ökonomie unmittelbar eine Antwort auf die Frage liefern, wie eine Bewahrung der Demokratie gegen marktbestimmte postdemokratische Tendenzen aussehen könnte. Bei den Lösungsformen einer gerechten oder moralisierten Ökonomie ist zusätzlich zu fragen, wie eine umgestaltete Ökonomie zur Sicherung der politischen Demokratie beitragen kann. Hier wird unterstellt, aber nicht nachgewiesen, dass auch Formen einer gerechteren Gestaltung der Ökonomie und ihrer Moralisierung dazu beitragen, die politische Demokratie aufrechtzuerhalten und postdemokratische Tendenzen abzuschwächen. 
rem normativen Gehalt ermäßigen oder diesen umdefinieren. Es wird sich im Verlauf der Argumentation zeigen, dass sich die Unterschiede zwischen der Sphäre der Transaktionen einerseits ${ }^{7}$ und der Sphäre der Organisationen bzw. der institutionellen Grundstruktur andererseits als höchst bedeutsam erweisen. Transaktionen, also Tauschprozesse und Zahlungsvorgänge, entziehen sich - so die Überlegung - prinzipiell sowohl der inneren Demokratisierung als auch einer inneren Sicherung ihres gerechten Gehalts. Eine Moralisierung von Transaktionen bietet dagegen keine Denkschwierigkeit, erscheint aber von den Realisierungsmöglichkeiten als die schwächste Variante der normativen Durchdringung. Auf der Ebene der Transaktionen ist zudem immanent eine Tendenz zur Ungleichheitsproduktion und zur Akkumulation der Ungleichheiten wirksam, die nicht selbst durch transaktionsinterne Regeln gesteuert oder aufgehalten werden kann. Sollten sich diese Überlegungen, die sich der Auswertung und Auseinandersetzung mit einzelnen Theorieansätzen verdanken, als hinreichend begründbar erweisen, muss sich eine politische Theorie des Marktes und der Ökonomie zentral mit zwei Unmöglichkeitsthesen und einer Unvermeidlichkeitsbehauptung auseinandersetzen:

- der These der Unmöglichkeit einer inneren Demokratisierung von freiwilligen Transaktionen,

- der These der Unvermeidlichkeit der Akkumulation von potenziell Ungleichheit erzeugenden Nebenfolgen in einem System freiwilliger Transaktionen,

- der These der Unmöglichkeit eines Systems von Transaktionsregeln für Transaktionen, das zur Vermeidung oder Internalisierung dieser Effekte führt.

\section{Demokratisierung der Ökonomie?}

Eine rechtfertigungsfähige Verfassung der Ökonomie kann unter demokratietheoretischen Prämissen der kollektiven Selbstbestimmung qua politischer Gleichheit nur eine solche sein, die das ökonomische Geschehen durchgehend demokratisiert. Damit wäre eine radikale Abkehr vom Trennmodell vollzogen: Wirtschaftliche Entscheidungen müssten normativen Ansprüchen entsprechen, die auch an politische Entscheidungen gestellt werden. Verlangt man von diesen, dass sie sich als demokratisch legitim erweisen, so wäre dies auch von ökonomischen Entscheidungen zu erwarten. Wirtschaftliche Entscheidungen müssten dementsprechend als kollektiv verbindliche Entscheidungen auf der Basis politischer Gleichheit unter Bedingungen gesicherter Pluralität verstanden werden können bzw. als solche institutionalisiert werden. Demokratietheoretisch wäre eine Theorie der Ökonomie nur als Übergang zur „Wirtschaftsdemokratie“ zu konzipieren. Dieser Begriff, bereits seit der Weimarer Republik Teil des politischen Vokabulars (Naphta-

7 Diese Sphärendifferenz gilt nicht nur für ökonomische Vorgänge, sie kann auch auf die Politik übertragen werden. In ökonomischen Kontexten wird von Transaktionen gesprochen als kleinteiligen, basalen Kommunikationsvorgängen. Der Meinungsaustausch in der Öffentlichkeit wäre ein Beispiel für solche basalen Kommunikationen in der Politik, bei denen zu prüfen wäre, ob sie sich prinzipiell den normativen Ansprüchen entweder von Demokratie-, Moral- oder Gerechtigkeitstheorie fügen könnten. Daraus folgt die weitergehende Problematik, ob man von einer „demokratischen Öffentlichkeit" nur im Sinne einer demokratiekompatiblen bzw. demokratieförderlichen Öffentlichkeit, aber nicht im Sinne einer demokratisierten Öffentlichkeit sprechen kann. 
li 1966), hat nur selten eine intensivere Ausarbeitung und konzeptionelle Fortbildung erfahren. Weite Teile der Arbeiterbewegung haben sich in Abkehr von realsozialistischen und orthodox-marxistischen Konzeptionen des Begriffs „demokratischer Sozialismus" bedient, ohne dass jedoch ein theoretisch ausgearbeitetes Modell einer wirtschaftsdemokratisch-sozialistischen Ökonomie leitend geworden wäre. Während in den späten 1960ern und frühen 1970er-Jahren die Forderung nach Demokratisierung aller gesellschaftlichen Bereiche (Vilmar 1973) auch Überlegungen zur demokratischen Reorganisation der Marktwirtschaft nach sich zog (Investitionslenkung im „Orientierungsrahmen '85“ der SPD, vgl. Oertzen et al. 1976), folgten in der Phase wirtschaftsliberaler Hegemonie seit den 1980 ern bis in die 2000er kaum theoretische Entwürfe einer ökonomischen Demokratisierung. Wirtschaftsdemokratie zielt auf eine innere Demokratisierung der Marktökonomie, impliziert daher Weitergehendes als das Modell einer sozialen Demokratie, die einen starken Sozialstaat mit starken Regulationen der Marktwirtschaft kombiniert - und erschien angesichts der neoliberalen Offensive gegenüber der erreichten sozialstaatlichen Demokratie als politisch fernliegendes und durch den Zusammenbruch des Realsozialismus vollkommen illusionär gewordenes Projekt. Eine der wenigen Ausnahmen in dieser Phase bilden die Arbeiten von John E. Roemer, der 1994 gegen den Zeittrend einen Entwurf für einen Marktsozialismus vorlegte (vgl. auch Elster u. Moene 1989; Schweickart 1993, 2012). Es kann daher nur in sehr begrenztem Umfang auf Vorarbeiten zurückgegriffen werden, wenn man eine Demokratietheorie von der Politik auf die Ökonomie übergreifen lassen will.

Aber ist die gesellschaftliche Produktion und Verteilung von Gütern und Dienstleistungen überhaupt in ein Geschehen zu überführen, das auf demokratischen Prozeduren beruht? Um die Frage der Demokratisierbarkeit einer Ökonomie - noch ganz unabhängig von deren Wünschbarkeit - zu beantworten, ist ein Begriff der Demokratie erforderlich, der abstrakter angelegt ist als ein Demokratieverständnis, das sich immer schon nur auf ein politisches System bezieht. In der Demokratietheorie wird Demokratie als Bezeichnung für das Zusammenspiel einer Vielzahl von Institutionen, Rechtsregeln und Strukturen verwendet. Der Demokratiebegriff ist auf der Ebene nicht einzelner Verfahren, sondern eines (meist staatlich verfassten) politischen Systems angelegt. Doch lässt sich bei Robert A. Dahl eine hinreichend abstrakte Definition der Demokratie finden, die als sphärenindifferente Bestimmung des Demokratischen fungieren könnte: "Democracy provides opportunities for: 1 . Effective participation, 2. Equality in voting, 3. Gaining enlightened understanding, 4. Exercising final control over the agenda, 5. Inclusion of adults" (Dahl 1998, S. 38). Die Definition macht vielleicht noch nicht hinreichend klar, dass die basale demokratische Leistung in der Schaffung einer Sphäre formaler Egalität jenseits der realen Ungleichheiten, also vor allem in der voting equality, besteht - in diesem Element ist die radikale Ablösung der Grundlagen kollektiver Entscheidung von allen sozialen Gegebenheiten und Vorstrukturierungen verkörpert. Vorausgesetzt ist in dieser Begriffsbestimmung zudem, dass die Angelegenheiten, die demokratisch entschieden werden sollen, Gegenstand eines Verfahrens kollektiv verbindlichen Entscheidens sein müssen. Entsprechend wird den folgenden Ausführungen ein Demokratie- und Demokratisierungsver- 
ständnis unterlegt, das politische Gleichheit im Sinne von Wahlgleichheit, Inklusion, effektiver Partizipation, Sicherung von Pluralität sowie kollektiv verbindliches Entscheiden umfasst. Damit ist eine Bestimmung von Demokratie gefunden, die begrifflich abstrakter angelegt ist als in jenen Theorien, die sich explizit auf politische Systeme und deren komplexe institutionelle Infrastruktur beziehen (z. B. liberale, republikanische, deliberative, partizipatorische, agonale Demokratietheorien) oder eine bestimmte institutionelle Verbindung zwischen Ökonomie und Politik schon als Bestandteil der Demokratietheorie postulieren (z. B. die Theorie sozialer Demokratie). Eine Wirtschaftsdemokratie kann auf der Basis dieser weiten Demokratiedefinition als eine von kollektiv verbindlichen Verfahren getragene ökonomische Ordnung verstanden werden, die Gleichheit in dem genannten Sinne zur Geltung kommen lässt.

Eine derartige Vorklärung des Begriffs Demokratie ${ }^{8}$ ist angesichts von prominenten Publikationen erforderlich, die den Demokratiebegriff in ökonomienahen Kontexten nutzen, aber darunter keineswegs eine innere Demokratisierung des Marktes oder der Marktwirtschaft verstehen. Zwei Beispiele seien hier genannt: So verbindet Robert J. Shiller, der die Krisen der 2000er-Jahre zutreffend prognostiziert hatte, mit dem Aufruf „democratize finance“ in seinem jüngst erschienenen Buch „Finance and the Good Society“ (Shiller 2012) einen Begriff der Demokratisierung, der darunter nur die erweiterte Nutzung beliebiger Instrumentarien durch immer weitere Teile der Bevölkerung versteht und Demokratisierung mit Konsumenteninklusion gleichsetzt. Als ein solches Instrumentarium nennt Shiller Finanzprodukte und Finanzinstitutionen. So lässt sich nach Shiller zeigen, dass die Nutzung von Versicherungen, Banken und anderen Finanzdienstleistern sich im historischen Verlauf von einem engen Zirkel wohlhabender Personen auf breiteste Teile der Bevölkerung erstreckt hat. Diese Vorgänge tauft Shiller „democratization of finance“. Der geschichtliche Rückblick dient der Vorbereitung der aktuellen politischen Empfehlung, trotz der Erfahrungen mit der Finanzindustrie in der Krise nicht in Feindschaft zu dieser Branche zu münden oder die Einführung neuer Finanzprodukte zu verhindern, sondern den Weg einer weiteren Demokratisierung zu beschreiten. Statt die Abhängigkeit von der Finanzindustrie zu verringern, plädiert Shiller für eine weitere Demokratisierung, also Ausdehnung dieses Sektors und weiterer Produktinnovationen auf möglichst alle Teile der Bevölkerung.

8 Auch die Begriffskombinationen aus Kapitalismus und Demokratie bedürfen einer genaueren Analyse. Richard A. Posner schreibt über „The crisis of capitalist democracy“ (2010), Wolfgang Streeck spricht dagegen über „Die vertagte Krise des demokratischen Kapitalismus“ (2013). In beiden Fällen ist ein Kapitalismus gemeint, der mit einer Demokratie koexistiert. Der demokratische Kapitalismus Streecks ist sicher nicht ein Kapitalismus, der die Eigenschaft besitzt, demokratisch zu sein, sondern ein Kapitalismus, der in demokratische politische Systeme eingebettet ist. Die Begriffswahl ermöglicht zwar, von einer „Entdemokratisierung des Kapitalismus“ (Streeck 2013, S. 28) seit den 1970er-Jahren zu sprechen, lässt dann aber als demokratische Alternative zur postdemokratischen Entwicklung nur die Rückkehr zu der Konstellation der 1950er bis 1970er zu. Der Begriff kapitalistische Demokratie als Spezifizierung der heutigen Ausprägung demokratischer Systeme als zunehmend marktgerechter (Post-) Demokratien besitzt dagegen eine höhere Sachgerechtigkeit und ein höheres Kritikpotenzial, auch wenn Posner eher kapitalismus-affin argumentiert. 
"Financial capitalism is an invention, and the process of inventing it is hardly over. The system has to be further expanded and democratized and humanized, so that we may reach a time when financial institutions will be even more persuasive and positive in their impact. That means giving people the ability to participate in the financial system as equals, with full access to information and with the resources, both human and electronic, to make active and intelligent use of their opportunities. It will mean that they truly consider themselves part of modern financial capitalism and not the victims of the aggressive and selfish acts of a cynical financial establishment" (Shiller 2012, S. vii-viii).

Auch der Begriff der „market democracy“, den John Tomasi in „Free Markets Fairness“ (2012) zur Kennzeichnung seiner politischen Theorie und eines Forschungsprogramms wählt, zielt nicht auf eine Demokratisierung der Marktökonomie im oben definierten Sinne. Als Hybrid aus Hayek und Rawls angekündigt, erscheint die Market Democracy als eine Nutzung der Rawlsschen Theoriesprache bei Einpflanzung eines Hayekschen Kerns aus starken ökonomischen (Eigentums- und Vertragsfreiheits-)Rechten samt deren verfassungsrechtlicher Garantie sowie rechtlich überprüfbaren bzw. einforderbaren Einschränkungen der staatlichen Interventionsmöglichkeit zur Redistribution (und damit zur Realisierung des Differenzprinzips). Den Nutzen und die Akzeptabilität dieses Systems für die Schlechtestgestellten soll der Marktprozess selbst gewährleisten. Das geschieht zum einen über die Wachstumseffekte einer freigesetzten Marktlichkeit, zum anderen über die Effekte auf die Selbstachtung der Individuen. Tomasi sieht in dem selbst verdienten (earned) und verdienten (deserved), nicht über Transfers erworbenen Einkommen die wichtigste Grundlage der Verbesserung der Lage der Schlechtestgestellten. Nur in individuellen Verhandlungen mit Arbeitgebern vereinbarte Löhne geben das Gefühl der Selbstautorenschaft seines eigenen Lebens. Sozialversicherung und umverteilende Steuern erscheinen ebenso wenig als sinnvolle Elemente der staatlichen Intervention wie Arbeitsschutz- und Mindestlohnregelungen. So bleibt nicht viel mehr als ein Hayeksches Programm von Sozialhilfe und öffentlicher Bildungsfinanzierung, die Marktdemokratie ist antiinterventionistisch und antietatistisch. John Tomasis Marktdemokratie ist mithin die programmatische Fortsetzung neoliberaler Politik im Gewande Rawlsscher Terminologie. Die Rolle kollektiver Lohnverhandlungen und staatlicher Programme als Grundlage eines Selbstgefühls, das gemeinsam Erkämpftes und die Effekte institutioneller Schutzregeln zu schätzen weiß, bleiben ebenso unbeachtet (Anderson 2013) wie die Steigerung sozialer Ungleichheit in einer derart freigesetzten Marktgesellschaft. Durch Stärkung der Eigentumsrechte als Teil des ersten Gerechtigkeitsgrundsatzes wird der zweite Gerechtigkeitsgrundsatz nur noch nominell angerufen, seine Realisierung durch den Selbstlauf des Marktes (statt durch Einrichtungen innerhalb der Grundstruktur) wird aber nur noch unterstellt. Zur demokratischen Qualität dieser Marktdemokratie lässt sich zudem nur wenig bei Tomasi finden. Er führt vielmehr vor, wie eine Strategie des Ausbaus der (Menschen-)Rechte gegen demokratische politische Autonomie in Stellung gebracht werden kann. Die stärkere Verankerung von Eigentumsrechten bedeutet, dass sich die Begründungspflichten für eine Einschränkung des privaten Freiheitsraumes 
deutlich erhöhen und gerichtlich detailliert überprüft werden können. Die Gesetzgebung soll durch eine strikte Rechtssprechung in ihrer Interventionsreichweite entscheidend eingeengt werden. Tomasis „Marktdemokratie“ ist ein konzeptioneller Entwurf zu einer durchgehend positiv bewerteten Postdemokratie, ein Manifest zur Abschaffung von politisch-demokratischen Steuerungsmöglichkeiten zugunsten privater Eigentumsrechte.

Dieser Versuch einer Begriffsverschiebung sollte Anlass geben, genauer danach zu fragen, was es überhaupt heißen kann, die Ökonomie zu demokratisieren. Vor aller Diskussion über Einzelheiten wirtschaftsdemokratischer Konzeptionen und über deren - sicherlich zweifelhafte - Realisierungschancen sollte gefragt werden, ob eine ökonomische Ordnung überhaupt als demokratische denkbar ist. Wir können uns Demokratie und Demokratisierung nur vorstellen für eine organisierte Einheit einer Vielzahl von Personen: einen Verein, eine Partei, einen Verband, eine Schule, einen Staat, eine internationale Institution. In einem weiten Sinne lässt sich Demokratie auch noch vorstellen für eine Gruppe von Menschen, eine Freundesgruppe, eine spontane Versammlung oder auch eine Familie. Immer muss es sich um einen Personenzusammenhang oder eine Organisation handeln, damit überhaupt die Möglichkeit der Implementation demokratischer Verfahren wie Beteiligung aller Mitglieder bei Wahlen, Einrichtung von Ämtern, Gremien und einer Entscheidungsfindung mittels (qualifiziertem) Mehrheits-, Einstimmigkeits- oder Konsensprinzip gegeben ist. Demokratische Entscheidungen sind nur als kollektive und für ein solches Kollektiv verbindliche Entscheidungen denkbar. Individuelle Entscheidungen, verstanden als Entscheidungen einer einzelnen Person, sind nicht demokratisierbar (aber durchaus rationalisierbar, moralisierbar). Ohne eine kollektive Größe, oft auch verfasst als Organisation, die ihre Angelegenheiten qua kollektiver Entscheidung regelt, kann es offenbar keine Demokratie geben. Eine Marktökonomie in ihrer Gesamtheit stellt aber keine Organisation dar (Hayek 2003). Sicherlich spielen Organisationen, insbesondere Unternehmen, in der Marktwirtschaft eine tragende Rolle. Aber die Ökonomie mit all ihren Milliarden Zahlungsvorgängen ist insgesamt weder eine Organisation noch eine Versammlung von Menschen. Märkte zeichnen sich dadurch aus, dass sich freiwillige Transaktionen mit einer Vielzahl von Teilnehmern vollziehen. Märkte sind zwar in öffentliche Institutionen eingebettet, sie selbst sind aber nur ein Netzwerk aus Transaktionen. ${ }^{9}$ Kennzeichnend für Märkte ist die Möglichkeit, Kaufakte in prinzipiell unendlicher Weise miteinander zu verkoppeln: Der Austausch von Gütern und Dienstleistungen aller Art über freie vertragliche Vereinbarungen zwischen den Interessenten ist das interaktive und gerade organisatorisch nicht verfasste Element, das Märkte und Marktwirtschaft ausmacht. Diese Eigenschaft kommt Märkten zu - ganz unabhängig davon, ob man dazu neigt, diese als rational zu heiligen oder nicht, ob man ihnen Gleichgewichtstendenzen zuspricht oder von ihrer Instabilität ausgeht (Frydman u. Goldberg 2011; Fox 2009).

9 Vgl. dazu die wirtschaftssoziologischen Arbeiten von Luhmann 1988, Beckert 1997, Fligstein 2001, Bevir u. Trentmann 2004, Beckert et al. 2007, Beckert u. Deutschmann 2009, Aspers 2011, Deutschmann 2012. 
Will man eine marktlich verfasste Ökonomie durchgehend demokratisieren, müsste man von individuellen Tauschakten gänzlich auf kollektive Entscheidungen umstellen, mithin eine tauschfreie Planwirtschaft errichten. Demokratische Legitimität kann ein Wirtschaftssystem erlangen, wenn zum einen Unternehmen im öffentlichen Eigentum sind bzw. von der Gesamtheit der Bürger verwaltet werden und zum anderen Transaktionen einer strikten demokratischen Außen- und Gesamtsteuerung unterliegen. Wer demokratische Legitimität in die ökonomische Sphäre übertragen will, wird sich daher mit Märkten als Vielheit von Kaufakten kaum arrangieren können. Die verschiedenen Realisierungsversuche sozialistischer Planwirtschaft waren dadurch gekennzeichnet, dass sie nie den Versuch einer demokratischen Verfassung der Ökonomie unternommen haben, sondern autoritär (oder gar totalitär) gesteuert wurden. Von den konstitutiven Elementen der Demokratie wurde nur einer, die kollektiv verbindliche Entscheidung, realisiert, nicht aber die politische Gleichheit, die effektive Partizipation bei gesicherter Pluralität. Wo eine Planwirtschaft entstanden ist, war diese eine autokratisch verfasste. Eine ,Plandemokratie‘ bzw. eine plandemokratisch verfasste Ökonomie ist daher selbst auf der Ebene der Theorie nicht vertreten. Grund dafür mag auch sein, dass die Planwirtschaft - unabhängig von ihrer demokratischen oder autoritären Verfassung - bereits in den 1930er-Jahren als technisch-ökonomisch nicht realisierbare Ordnung angesehen wurde. In der Debatte zwischen Oskar Lange und Friedrich Hayek (siehe Roemer 1994, S. 28-36) erschien die Planwirtschaft als informationstechnische Unmöglichkeit. Lange hatte die Planwirtschaft aber als Tâtonnement-Prozess beschrieben, womit er der Walrasianischen Markttheorie noch verbunden blieb, an die Stelle des Auktionators allerdings das zentrale Planungsbüro setzte. Der Plan war mithin eher eine Vorab-Simulation eines Marktgeschehens, eine Art Ex-Ante-Markt. Eine gesellschaftliche Deliberation, aus der der Plan als kollektive demokratische Entscheidung hervorging, wurde nicht erörtert.

Kapitalismuskritiker, die den realsozialistischen Autokratien ablehnend gegenüberstanden, wandten sich entsprechend Konzepten eines marktwirtschaftlichen Sozialismus zu. Damit wurde anerkannt, dass die Ebene der einzelnen Transaktionen und ihrer Vernetzung, die Ebene der Tauschprozesse und Zahlungsvorgänge, nicht ersetzt oder intern auf einen grundlegend anderen Modus umgestellt werden kann. Dieser Literatur scheint theoretisch nicht vorstellbar, wie Käufe und Zahlungen als Transaktionsformen demokratisiert werden sollten. Es gibt danach Grenzen der Demokratisierbarkeit einer Marktökonomie: Dort, wo es um Organisationen geht, lassen sich demokratische Verfahren implementieren, und auch dort, wo Tauschakte reguliert werden sollen, lassen sich die Regulierungsformen und -institutionen demokratischen Zuschnitts denken. Das Projekt einer wirtschaftlichen Demokratisierung lässt sich also durchaus ein erhebliches Stück vorantreiben. Allein in den basalen Transaktionsformen wie Zahlung und Tausch und den durch sie gebildeten „Spontanbereichen“ (Teubner 2012, S. 140), den Märkten, sind Grenzen für die Anwendbarkeit demokratischer Verfahren und damit Grenzen der Expansion demokratischer Legitimität gesetzt. Eine Marktwirtschaft kann in all ihren Grundelementen nicht zu einer demokratischen Ordnung werden. Daraus ergibt sich eine nicht hintergehbare Differenz zwischen Markt- 
ökonomie und demokratischer Politik. Ökonomische Tauschprozesse sind auf der Ebene der Transaktion nicht demokratisierbar: Es kann hier keine formale Stimmengleichheit geben. Demokratisierung verlangt die Überwindung der realen Ungleichheiten (wie der der Verfügung über Finanzmittel oder andere Ressourcen) durch die Schaffung einer künstlichen Ebene der Gleichheit, der Stimmengleichheit. Es ist nicht ersichtlich, wie Tauschvorgänge von der Ebene der Zahlungsfähigkeitsunterschiede auf die Ebene der Stimmengleichheit überführt werden könnten. Die genuin marktliche Transaktion entzieht sich einer Umstellung auf den Modus demokratischen Entscheidens. Man kann sich mithin eine Demokratie ohne Tauschvorgänge vorstellen, aber keine genuin demokratische Tauschund Marktökonomie.

Diese Überlegungen machen vielleicht auch verständlich, warum sich die Diskussion um industrielle Demokratie bzw. Wirtschaftsdemokratie vor allem auf die Frage des Eigentums und der Steuerung von Unternehmen verlegt hat: Unternehmen sind demokratisierbar. Über die deutsche Form der Mitbestimmung hinausgehend werden Modelle der Arbeiterselbstverwaltung, der von Managern geführten Unternehmen mit strikten Regeln für die Einkommensverteilung sowie öffentliche Eigentumsmodelle verschiedener Art diskutiert (Roemer 1994, S. 4654; Demirovic 2007). ${ }^{10}$ Der Theoretiker, auf dessen Demokratiedefinition sich dieser Aufsatz stützt, hat in Fortführung von Überlegungen, die bereits in Dahl u. Lindblom 1953 angelegt waren, mit dem Buch „A Preface to Economic Democracy “ im Jahre 1985 einen Entwurf einer solchen konsequenten Demokratisierung von Unternehmen vorgelegt: Dem „corporate capitalism“ stellt er durch die Betriebsangehörigen selbstverwaltete Unternehmen auf der Basis kooperativen Eigentums gegenüber. Doch jenseits der inneren Demokratisierung der Unternehmen bleibt auch bei ihm der nicht demokratisierte Markt.

\section{Wenn nicht demokratisch, dann doch gerecht?}

Im Rahmen der Demokratietheorie scheint es keine Möglichkeit zu geben, eine normgerechte Lösung für die Transaktionsebene der Ökonomie anzubieten. Tauschprozesse lassen sich nicht intern demokratisieren, nicht auf die normative Grundlage politischer Gleichheit stellen. Deshalb wird im Folgenden zu prüfen sein, ob sich ökonomische Transaktionssysteme gerecht gestalten lassen und mit der Vorstellung einer gerechten Gesellschaft kompatibel gemacht werden können. Dazu wird die Rawlssche Gerechtigkeitstheorie als wirkungsmächtigste und wohl auch am stärksten systematisierte Theorie insbesondere in ihrer Auseinanderset-

10 Wird die Unternehmensebene verlassen, geht es um demokratische Formen unternehmensübergreifender Mitbestimmung in Branchen, auf nationalen Märkten und bei globalen Investitionsentscheidungen. Demokratisierbarkeit ist dort aber nur gegeben, wenn es um Regulierungsgremien geht - z. B. in Überlegungen zur demokratischen Umgestaltung bestehender korporatistischer Arrangements auf Branchenebene oder zur Einführung Dritter Kammern als Arbeits- und Wirtschaftskammern. 
zung mit libertären Entwürfen befragt. ${ }^{11}$ Letztere stellen vertragsförmige Tauschprozesse ins Zentrum ihrer Thematisierung von Gerechtigkeit. In den Theorien von John Locke und Robert Nozick bilden miteinander verkettete Transaktionen, die als in sich gerecht angesehen werden können, die Basis, um alle daraus resultierenden gesellschaftlichen Zustände als gerecht zu bezeichnen. Im Sinne reiner Verfahrensgerechtigkeit ${ }^{12}$ wird in diesen Theorien angenommen, dass ausgehend von einem gerechten Ausgangszustand und einer Folge von in sich gerechten Transaktionen jeder beliebige gesellschaftliche Zustand, der sich aus dieser Verkettung ergibt (solange die Gerechtigkeit jedes einzelnen Schrittes eingehalten ist), als gerecht oder legitim gilt. Es gibt mithin keine Auszeichnung eines spezifischen Verteilungszustandes als gerecht: Letztlich könnte jede beliebige Verteilung als gerecht gelten, wenn sich nachweisen ließe, dass sie ausschließlich aus fairen Transaktionen und einer fairen Ausgangsverteilung hervorgegangen ist. Die Basis dieser Konstruktion ist die einzelne Transaktion, die unabhängig von allen gesellschaftlichen Institutionensystemen als gerecht oder ungerecht bezeichnet wird (zur Debatte über institutionsunabhängige Gerechtigkeitsmaßstäbe vgl. Olsaretti 2003). Freiwillig eingegangene Vertragsbeziehungen, verstanden als Äquivalententausch unter Ausschluss von Gewalt, Bedrohung, Täuschung und Informationsasymmetrien, gelten als kleinste (gerechtigkeitstheoretisch nicht kritisierbare) Bausteine einer Theorie der gerechten Verfassung und Entwicklung einer Gesellschaft (Nozick 1974). Wenn diese Argumentation aber zutreffen sollte, wäre eine reine Marktökonomie als gerecht zu legitimieren, unabhängig von ihren Folgen für die demokratische Verfasstheit politischer Entscheidungen und für den Grad sozialer Ungleichheit einer Gesellschaft.

Eine derart marktaffine Gerechtigkeitstheorie lässt sich nur angreifen, wenn entweder die Grundannahme gerechter Transaktionen bezweifelt wird, oder die Annahme verworfen wird, dass aus gerechten Transaktionen gerechte gesellschaftliche Verhältnisse folgen. John Rawls nutzt in „Die Grundstruktur als Gegenstand“ (Rawls 1998, S. 367-403) überwiegend die zweite Kritikstrategie. Rawls bezweifelt zwar die Erkennbarkeit der inhärenten Gerechtigkeit freiwilliger Transaktionen bei Fehlen einer gerechten Grundstruktur:

„Erstens können wir nicht durch bloßes Betrachten des Verhaltens von Individuen und Vereinigungen in ihrer unmittelbaren (oder lokalen) Umgebung entscheiden, ob

11 Eine Auseinandersetzung mit der Perspektive anderer bekannter Gerechtigkeitstheorien auf Markt und Ökonomie kann an dieser Stelle nicht geleistet werden. Während Walzers komplexe Theorie der Gleichheit von „an art of differentiation“ (Walzer 1983, S. XV) bestimmt wird und daher für die hier gestellte Frage einer Ökonomie und Politik übergreifenden Theoriebildung wenig Ansatzpunkte bietet, ist eine nähere Betrachtung z. B. der Dworkinschen Konzeption von Ressourcengleichheit mit den Elementen Neidtest, Auktion und Versicherung auch deshalb erforderlich, weil sie wesentlich marktfreundlichere Schlussfolgerungen auf einer egalitaristischen Basis zu gestatten hofft (vor allem Dworkin 2000 [Auszüge dt. 2011b und 2011a: S. 351-363]). Ganz andere Herausforderungen stellt die Analyse von Amartya Sens Gerechtigkeitstheorie (2009) aufgrund ihrer Abkehr von einem idealtheoretischen Vorgehen.

12 Rawls unterscheidet drei Formen der Verfahrensgerechtigkeit: vollkommene, unvollkommene und reine. Letztere definiert er wie folgt: „Im Gegensatz dazu liegt reine Verfahrensgerechtigkeit vor, wenn es keinen unabhängigen Maßstab für das richtige Ergebnis gibt, sondern nur ein korrektes oder faires Verfahren, das zu einem ebenso korrekten oder fairen Ergebnis führt, welcher Art es auch sei, sofern das Verfahren ordnungsgemäß angewandt wurde.“ (Rawls 1979, S. 107). 
die erreichten Übereinkünfte - aus einer gesellschaftlichen Perspektive betrachtet gerecht und fair sind, denn eine solche Einschätzung hängt entscheidend von den Eigenschaften der Grundstruktur ab, nämlich davon, ob es ihr gelingt, die Hintergrundgerechtigkeit zu erhalten“ (Rawls 1998, S. 377).

Aber er unterstellt für die weitere Argumentation, dass sich die Behauptung der inhärenten Gerechtigkeit von freiwilligen Tauschakten belegen lasse.

\subsection{Die Unmöglichkeit eines gerechten Transaktionssystems}

Das Locke-Nozick-Argument ist zunächst durchaus einsichtig: Gegeben eine offene Ausgangssituation (keinerlei Eigentumszuordnung) und ein System von fairen Regeln für die Aneignung von Besitz (principle of justice in acquisition), ein System von fairen Regeln für die Transaktion von Besitz (principle of justice in transfer, darunter auch Schenkungen), Regeln für die Korrektur von Verfehlungen der beiden ersten Prinzipien (principle of rectification) sowie die Möglichkeit der Bildung von privaten Vereinigungen (auch der Staat ist in diesem System nichts Anderes als eine private Vereinigung), ist bei Einhaltung dieser Regeln der Schluss auf faire Verteilungen zu jedem beliebigen Zeitpunkt schlüssig (Nozick 1974, S. 150-160). Rawls‘ zentrales Gegenargument lautet (vgl. auch Maffetone 2010, S. 173-177): Selbst wenn alle Transaktionen aus einem gerechten Naturzustand korrekt und fair erfolgen würden, seien die Ergebnisse doch nicht fair, da die $\mathrm{Ku}-$ mulation der Transaktionen so viele Nebenfolgen mit sich bringe, dass die Hintergrundgerechtigkeit untergraben wird.
„Für die Bewahrung der Hintergrundgerechtigkeit reicht es nicht aus, wenn alle Ver- nünftigen darin übereinstimmen, es werde fair gehandelt, und alle hielten sich pein- lich genau an die Regeln. Dies ist ein wichtiger, offensichtlicher Punkt. Wenn unsere soziale Welt von Unaufrichtigkeit und Täuschung durchdrungen ist, liegt es nahe, zu glauben, Gesetze und Regierungen seien nur wegen der Neigung der Individuen zu unfairem Handel notwendig. Die Tendenz geht jedoch eher dahin, dass die Hinter- grundgerechtigkeit selbst dann untergraben wird, wenn die Individuen fair handeln. Die kumulativen Resultate der einzelnen voneinander unabhängigen Transaktionen entfernen sich vom Zustand der Hintergrundgerechtigkeit und nähern sich ihm nicht an. Wir können sagen, die unsichtbare Hand weise hier in die falsche Richtung und begünstige oligopolistische Akkumulationen, die zur Stabilisierung ungerechtfertigter Ungleichheiten und Beschränkungen fairer Chancen führten. Deshalb benötigen wir spezielle Institutionen zur Wahrung der Hintergrundgerechtigkeit und eine besondere Gerechtigkeitskonzeption, die festlegt, wie diese Institutionen eingerichtet werden müssen“ (Rawls 1998, S. 378).

Rawls verneint die Möglichkeit einer Entwicklung, die auf der Basis gerechter Interaktionen gerechte Verteilungen erlaubt. Das ist ein entscheidendes Argument gegen die Locke/Nozicksche Variante der genealogischen reinen Verfahrensgerechtigkeit, allerdings geleitet von einem gegenüber dem Locke-Nozickschen Denkansatz externen Kriterium gerechter Verteilungen. Kausal ausschlaggebend sind für Rawls Nebenfolgen, die auch bei strikter Einhaltung der fairen Verfahren auf der Basis einer fairen Ausgangsverteilung eintreten. Warum eine Folge aus 
fairen Ausgangsbedingungen und fairen Transaktionen zu einem hohen Maß an Oligopolisierung und Ungleichheit führt, erklärt Rawls ansatzweise: Es seien die kumulativen Resultate vieler einzelner Vereinbarungen, „, verbunden mit sozialen Tendenzen und historischen Zufälligkeiten “, die auf Dauer die Voraussetzungen fairer Übereinkünfte zerstören (Rawls 1998, S. 376). Nennen wir dies die These der unvermeidbaren Nebenfolgen fairer Transaktionen.

Ohne noch weitere Erklärungen über die Funktionsweise dieser Kumulation abzuliefern, verstärkt Rawls seine Kritik durch eine zweite These, die These der Unmöglichkeit von handhabbaren Regeln, die Abweichungen von einer historischen Kette rein fairer Transaktionen ausschließen:

„Es gibt keine handhabbaren Regeln, die diese unerwünschten Konsequenzen ausschlössen und deren Befolgung man den wirtschaftlich Handelnden bei ihren täglichen Transaktionen auferlegen könnte. Die Konsequenzen liegen oft so weit in der Zukunft oder sind so indirekt, dass der Versuch, ihnen durch einschränkende Regeln vorzubeugen, für die Individuen eine übermäßige, wenn nicht untragbare Belastung bedeuten würde“ (Rawls 1998, S. 377).

Dass keine „formulierbaren und praktikablen Regeln“ existieren können, um das Untergraben der Hintergrundgerechtigkeit zu verhindern, liege daran,

„dass die für Vereinbarungen und individuelle Transaktionen geltenden Regeln nicht zu komplex sein sollten und zu ihrer richtigen Anwendung nicht zu viele Informationen erfordern dürfen. Auch sollten Individuen nicht mit weit verstreuten dritten Parteien verhandeln müssen, denn dies würde ihnen zu hohe Transaktionskosten auferlegen. Schließlich sind die für Vereinbarungen geltenden Regeln öffentliche Handlungsvorschriften und keine mathematischen Funktionen, die so kompliziert sein dürfen, wie man es sich nur vorstellen kann“ (Rawls 1998: S. 378).

Prinzipiell wäre es also denkbar, die Nebenfolgenbewältigung in die Transaktionsregeln (also das Privatrecht) einzubauen, was aber einen sehr hohen Informationsaufwand angesichts der weit von dem Ort und dem Aktionsfeld der eigentlichen Transaktion entfernten und weit in der Zukunft liegenden Konsequenzen bedingen würde. Dies wäre, so darf man Rawls verstehen, vielleicht im Rahmen eines komplexen Algorithmus zu lösen, nicht aber im Rahmen klarer und handhabbarer, öffentlich verstehbarer und implementierbarer Regeln. Es gibt also pragmatische, aber doch sehr grundlegende Hindernisse in der Detaillierung der Transaktionsregeln. Nennen wir dies die These der prinzipiellen Grenzen der internen Regulierung von Transaktionen.

Da Nebenfolgen und Kumulationen von Nebenfolgen immer eintreten und diese durch Regelsysteme niemals zu vermeiden sind, kann auch niemals ein stabiler gerechter Zustand allein über ein System von Interaktionen und Regeln für diese Interaktionen gesichert werden. Entwicklungen, die allein auf Systemen und Verkettungen fair geregelter Transaktionen beruhen, sind grundlegend instabil und führen langfristig zur Steigerung von Ungleichheiten und zu Oligopolen, unabhängig davon, wie sehr die Regelsysteme für Transaktionen verfeinert werden. Systeme von Transaktionsregeln führen nicht zu spontanen Ordnungen, sondern zu sich steigernden Ungleichverteilungen und können daher nicht als Kandidat 
für reine Verfahrensgerechtigkeit in Betracht kommen. Die Abwehr des LockeNozick-Modells reiner Verfahrensgerechtigkeit arbeitet allerdings mit einem externen Kriterium für angemessene Verteilungsergebnisse. Rawls setzt voraus, dass es Zustände gibt, die nicht akzeptabel sind: Oligopole, dauernde Verletzungen der Chancengerechtigkeit oder ausgeprägte soziale Ungleichheit. Nur unter Heranziehung eines solchen Outcome-orientierten Kriteriums lässt sich zeigen, dass die Einhaltung von Regeln fairer Transaktion bei fairer Ausgangslage nicht akzeptabel ist. So findet keine rein immanente Widerlegung des Locke-Nozickschen Ansatzes statt, denn dieser beanspruchte, dass es jenseits der Einhaltung von Regeln der Aneignung und des Tausches keine Maßstäbe des Gerechten gebe, mithin jede auf gerechtem Tausch beruhende Verteilung gerecht sei, möge sie von Ungleichheiten größeren Ausmaßes gekennzeichnet sein oder nicht.

\subsection{Grundstruktur und Transaktionsregeln}

Rawls schließt aber aus der Unmöglichkeit, eine Gerechtigkeitstheorie auf der Idee gerechter Interaktionen bzw. fairen Tausches zu gründen, dass die primäre Aufgabe der Gerechtigkeitstheorie die Einrichtung einer institutionellen Grundstruktur einer Gesellschaft sei. Statt von Interaktionen und Tauschketten als sozialen Mikroprozessen baut Rawls die Gerechtigkeitstheorie von der gesellschaftlichen Makrostruktur her auf. Innerhalb des von der Grundstruktur gesetzten Rahmens, der die Hintergrundgerechtigkeit sichert, spielen sich natürlich Interaktionen der unterschiedlichsten Art ab, die aber so institutionell vorstrukturiert und reguliert sind, dass die Gerechtigkeit des gesellschaftlichen Zusammenspiels erhalten bleibt. Die Institutionen (Grundstruktur) sind so stark strukturierend, dass das freie, Nebenfolgen erzeugende Spiel der Interaktionen (Tauschprozesse) die Gesamtgerechtigkeit des gesellschaftlichen Systems nicht zerstören kann.

In Anwendung der Überlegungen zur Nozickschen Theorie wird aber zu fragen sein, ob die Grundstruktur einem ,Stresstest', wie ihn Rawls für die Transaktionsgerechtigkeit entworfen hat, in analoger Weise standhalten würde. Zwar fand die Frage der Stabilität einer auf Gerechtigkeitsprinzipien gegründeten, einer wohlgeordneten Gesellschaft, stets hinreichend Beachtung in der Rawlsschen Theorie. Die zwei Thesen gegen Nozick können jedoch als Leitfaden für eine gesonderte Prüfung genutzt werden: Wie kann man sich die Bewältigung ökonomischer Transaktionsdynamiken in einer gerechten Gesellschaft vorstellen? Kann verhindert werden, dass auch bei Einrichtung einer Grundstruktur strikt nach den beiden Gerechtigkeitsprinzipien permanent Nebenfolgen auftreten, die sich kumulieren und zu Ungleichgewichten führen? Und lässt sich ein hinreichend detailliertes System von institutionellen Vorkehrungen innerhalb der Grundstruktur entwerfen, sodass Nebenfolgen eingefangen und korrigiert werden können? Und gelingt es, dieses Regelsystem so zu entwickeln, dass es nicht so kompliziert ausfällt und so viele Informationen berücksichtigen muss, dass es nicht mehr als verständliche öffentliche Handlungsrichtlinie, sondern nur noch als Algorithmus denkbar ist? Sollten diese Fragen verneint werden müssen, wäre auch die Rawlssche Konstruktion einer reinen Verfahrensgerechtigkeit für die Grundstruktur hinfällig und wir 
könnten nicht beliebige, von den Institutionen der Grundstruktur erzeugte Verteilungszustände als gerecht auszeichnen.

Auch in einer wohlgeordneten Gesellschaft findet sich eine Ungleichheiten erzeugende Dynamik. Die erste These der unvermeidlichen Nebenfolgen fairer Tauschakte gilt sicherlich auch innerhalb einer gerechten Gesellschaft. Diese Nebenfolgen werden jedoch - anders als bei Nozick - von einer selbst Gerechtigkeit verkörpernden Grundstruktur reguliert und korrigiert: „So besteht die Grundstruktur zunächst aus denjenigen Institutionen, welche die gesellschaftlichen Rahmenbedingungen festlegen, und schließt Maßnahmen ein, welche die unvermeidlichen, der Hintergrundgerechtigkeit zuwiderlaufenden Tendenzen fortwährend berichtigen und ausgleichen, wie die Besteuerung von Einkommen und Erbschaften, die dazu dient, Besitzstände einander anzugleichen " (Rawls 1998, S. 379). In die Grundstruktur sind Maßnahmen eingebaut, die die Abweichung von den Gerechtigkeitsprinzipien, die auch innerhalb der institutionellen Regeln auftreten, korrigieren und im Sinne des Differenzprinzips Einkommens- und Vermögensumverteilungen herbeiführen. Ist die Grundstruktur gemäß den Gerechtigkeitsgrundsätzen entfaltet, sind damit alle daraus resultierenden Verteilungen gerecht (vgl. Freeman 2007, S. 126). Können aber die zur Grundstruktur zählenden Maßnahmen hinreichend detailliert und langfristig geltend bestimmt werden? Oder handelt es sich letztlich um Ad-hoc-Maßnahmen, die immer dann getroffen werden müssen, wenn Abweichungen erkannt werden (was wiederum ein externes Kriterium für Verteilungszustände voraussetzte) - und die daher auch immer anders ausfallen werden?

Gegen Nozick hatte Rawls auf der Unmöglichkeit angemessener korrigierender Regeln bestanden. In seiner Gerechtigkeitstheorie der institutionellen Verfassung einer Gesellschaft sind korrigierende Regeln wie Steuer- und Erbschaftsgesetze Teil einer wirksamen Grundstruktur. Offensichtlich sind für Rawls verteilungskorrigierende Regeln im Fall der Grundstruktur möglich, im Fall der internen Regulierung von Transaktionen unmöglich. Unmöglich sind für Rawls Regulierungen, die in die Transaktionsregeln (das Privatrecht) eingelassen sind. Dagegen sind öffentliche Regelsetzungen möglich, die jenseits der einzelnen Tauschakte ansetzen und die Folgen einer Folge von Tauschakten in ihren Wirkungen konterkarieren, z. B. durch stark redistributiv wirkende Steuergesetze. Während der Ansatzpunkt des Tauschaktes oder Vertrages verlangt, im einzelnen Vertrag auf rechtlich verbindliche Weise alle jene Folgen einzukalkulieren und auszugleichen, die als Ungleichheitsträger gelten könnten, verlangt eine öffentlich-rechtliche Regulierung nur die Beobachtung der Aggregatdaten, der Einkommens- und Vermögensentwicklung und einen summarischen Ausgleich. Der einzelne Zahlungsvorgang, der jeweilige Vertrag, wird nicht selbst zum Regulierungsobjekt. Die Grundstruktur der Rawlsschen Gerechtigkeitstheorie muss daher eine Art Ungleichheit selbständig korrigierende Maschinerie sein, die anhand von Ungleichheitsdaten die angemessene Restrukturierung der individuellen Einkommens- und Vermögenspositionen vornimmt, ohne direkt in die einzelnen Verträge und Tauschakte einzugreifen. Rawls reagiert auf diese Problematik mit der Unterschei- 
dung von zwei Arten von Regeln, ${ }^{13}$ von denen die eine zur Grundstruktur zählt, die andere nicht - eine Differenzierung, die Rawls als institutionelle Arbeitsteilung bezeichnet:

\begin{abstract}
„Zusammengefasst: Wir gehen von der Grundstruktur aus und versuchen uns klarzumachen, wie diese Struktur selbst die zur Bewahrung der Hintergrundgerechtigkeit notwendigen Anpassungen vornimmt. Was wir letztlich anstreben, ist eine institutionelle Arbeitsteilung zwischen der Grundstruktur und den Regeln, die unmittelbar für Individuen und Vereinigungen gelten und von ihnen bei einzelnen Transaktionen beachtet werden müssen. Ist diese Arbeitsteilung einmal etabliert, können Individuen und Vereinigungen ihre Ziele im Rahmen der Grundstruktur wirksamer verfolgen, in der Gewißheit, dass anderswo im gesellschaftlichen System die notwendigen Korrekturen zur Bewahrung der Hintergrundgerechtigkeit vorgenommen werden“ (Rawls 1998, S. 379-380, vgl. Schaub 2009, S. 139-141).
\end{abstract}

Die Transaktionsregeln wären überlastet mit der Aufgabe, gerechte Zustände herzustellen, zu wahren oder wiederherzustellen, deshalb bedarf es einer Grundstruktur, diese ständig erforderlichen Korrekturen bzw. Anpassungen („,adjustments“) vorzunehmen. Es sind vor allem die Maßnahmen der Steuer- und Wirtschaftspolitik, die diese Aufgabe innerhalb der Grundstruktur übernehmen.

Diese Regeln müssen aber den Anforderungen reiner Verfahrensgerechtigkeit unterliegen, sie sind nicht auf eine bestimmte Verteilungsstruktur ausgerichtet, sie reagieren nicht auf Ungerechtigkeiten, sondern folgen allein dem Differenzprinzip ohne Ansehung der jeweiligen Verteilungssituation. Das macht Rawls noch einmal sehr deutlich im $\mathbb{S} 9$ seines Aufsatzes „Die Grundstruktur als Gegenstand“, der die Notwendigkeit einer Idealform für die Grundstruktur verteidigt. Diese Anpassungen innerhalb der Grundstruktur besitzen den Charakter öffentlichen Rechts („public law“), sie gelten mithin nicht für die Transaktionen (Privatrecht), sie sind keine diskretionären Eingriffe in einzelne Transaktionen, Ansprüche werden nicht missachtet oder willkürlich außer Kraft gesetzt (Rawls 1998, S. 396). Aber wie sollte es solchen auf Dauer gestellten Steuer- und Wirtschaftspolitiken gelingen, immer die gerade erforderlichen Anpassungen gegenüber dem Transaktionsgeschehen vorzunehmen, wo doch die Nebenfolgen so zahlreich und unübersichtlich sind? Wie sollten sie zweitens fähig sein, sich dem Zirkel immer weiterer Detailliertheit und Unübersichtlichkeit zu entziehen, und wie sollte es drittens gelingen, genau die richtigen Anpassungen vorzunehmen, wenn man nur das Differenzprinzip, aber nicht die konkreten Verteilungslagen betrachten kann (womit man allerdings ein Kriterium zusätzlich zum Differenzprinzip einführen und von reiner auf unvollkommene Verfahrensgerechtigkeit umstellen würde, vgl. Nullmeier 2000)? Die Leistungskraft des öffentlichen Rechts gegenüber dem Privatrecht für Transaktionen wird von Rawls als recht hoch eingeschätzt, ohne dass er

13 Wollte man weiter differenzieren, so lassen sich bei Rawls zwei Regelgruppen mit je zwei Regeltypen finden: $\mathrm{Zu}$ den Regeln der Grundstruktur zählen Regeln, die die gesellschaftlichen Rahmenbedingungen festlegen, aber auch die Maßnahmen, die der Hintergrundgerechtigkeit zuwiderlaufende Entwicklungen korrigieren. Zu der zweiten Regelgruppe gehören die Regeln für Transaktionen und Vereinbarungen zwischen Individuen und Vereinigungen sowie die Regeln, die den Transaktionsregeln zur Durchsetzung verhelfen bzw. in Fällen der Regelverletzung (Betrug etc.) greifen. 
dafür Belege liefern würde. Es fällt leicht Rawls darin zu folgen, dass es solcher öffentlichen Gesetze zwingend bedarf, um Anpassungen zu ermöglichen, es fällt aber schwer anzunehmen, dass sie um so vieles perfekter sein sollten als Lösungen, die direkt am Vertragsrecht ansetzen. Vor allem ist schwer nachzuvollziehen, wie sich eine ständige Anpassungstätigkeit der Steuergesetze, der Finanz- und Wirtschaftspolitik ohne Betrachtung der jeweiligen Verteilungsstrukturen bewerkstelligen lassen soll. Wenn die Nebenfolgen der Transaktionen so unübersichtlich und weitgestreut ausfallen sollten wie von Rawls behauptet, dann wäre eine im Sinne des Differenzprinzips angemessene gesetzgeberische Anpassung durchaus auf ein Wissen über die jeweilige Gesamtlage der akkumulierten Nebenfolgen angewiesen. Sie müsste dieses aber direkt beurteilen können, was ein Zusatzkriterium über die Gerechtigkeit von Verteilungsstrukturen erforderte - was Rawls gerade ausschließen will. Allerdings könnte man aufgrund einer Rawls-Passage, die Green (2013) jüngst in einem Aufsatz zum entschuldbaren Neid hervorgehoben hat, ${ }^{14}$ schließen, dass die Ausrichtung der Anpassungen den Gerechtigkeitsgrundsätzen und ihren Stabilitätsbedingungen folgen muss, zu denen die Vermeidung entschuldbaren Neids gehört. Entschuldbar ist Neid, wenn er sich auf übergroße Ungleichheiten bezieht. Damit wäre doch ein Kriterium der Verteilungsstruktur in die Argumentation eingebaut worden, die Entwicklung der Grundstruktur als reiner Verfahrensgerechtigkeit partiell durchbrochen. Auch wenn Rawls in diesem Text davon spricht, dass man sich „weitgehend“ auf reine Verfahrensgerechtigkeit verlassen will (Rawls 1998, S. 394; „on a large element of pure procedural justice“ Rawls 1993, S. 281), würde er wohl nicht so weit gehen, ein unabhängiges Kriterium für Verteilungszustände zuzulassen. Wie es aber gelingen kann, die Folgen der nicht selbst auf die Gerechtigkeit der Gesamtgesellschaft ausgerichteten Transaktionen so aufzufangen, dass diese ständig gesichert ist, wird nicht vollständig geklärt. Letztlich will Rawls eine Privatrechtsgesellschaft fortbestehen lassen und nur Rahmenbedingungen so ändern, dass deren negative Folgen jederzeit eingefangen werden können.

\subsection{Ungerechtigkeitstheorie, Nicht-Beherrschung und Privatrecht}

In einem anderen Theorierahmen lässt sich aber zeigen, dass man die durch das Privat- bzw. Vertragsrecht, also die Regeln für Transaktionen, gesetzten Spielräume durchaus als das Element von Beherrschung ansehen kann, das einer Gerechtigkeitstheorie als Skandal gelten muss. Dazu wird hier auf die im direkten Anschluss an Philip Pettit (1999) von Frank Lovett (2010) formulierte Theorie der Gerechtigkeit als Minimierung von Beherrschung rekurriert. Dabei geht es weniger um eine Darstellung dieser Theorie als um den durchaus gegen die explizite

14 „Die beiden Grundsätze bestimmen auch eine Idealform für die Grundstruktur, von der ausgehend die laufenden institutionellen Prozesse und Verfahren eingeschränkt und angepasst werden. $\mathrm{Zu}$ diesen Einschränkungen gehören Begrenzungen der Akkumulation von Eigentum (besonders dann, wenn es Privateigentum an Produktionsmitteln gibt), die sich aus den Erfordernissen des fairen Wertes der politischen Freiheiten und fairer Chancengleichheit ergeben, und Begrenzungen aus Gründen der Stabilität und des entschuldbaren Neides, wobei beide Arten von Begrenzungen mit dem wesentlichen Grundgut der Selbstachtung zusammenhängen.“ (Rawls 1998, S. 397-398) 
Argumentation Lovetts gerichteten Versuch, die herrschafts- und gerechtigkeitstheoretische Problematik der tradierten Transaktionsregeln aufzuzeigen. Beherrschung (domination) wird bei Lovett wie folgt definiert: "In plain English, we can say that persons or groups are subject to domination to the extent that they are dependent on a social relationship in which some other person or group wield arbitrary power over them" (Lovett 2010, S. 119). ${ }^{15}$

Hier ist nur zu klären, ob marktbestimmte ökonomische Ungleichheiten unter den Begriff der domination fallen könnten, den Lovett verwendet. Traditionell gilt ein vollkommener Markt als System rein freiwilliger Beziehungen und ist damit kein Fall von Beherrschung. Lovett setzt seine Analyse auf der Ebene der einzelnen sozialen Beziehungen zwischen Personen und Gruppen an und hat daher für komplexe Vernetzungsstrukturen wie Märkte und auch für ökonomische Ordnungen insgesamt keinen Blick. Kapitalismus als Herrschaftsform taucht bei Lovett nicht auf. Märkte zeichnen sich dadurch aus, dass sie sich aus freiwilligen Transaktionen konstituieren und somit kein offensichtliches Beherrschungselement existiert. Zugleich ist die Marktsphäre diejenige der Freisetzung von Willkürfreiheit. Eine marktkritische Sicht würde nun behaupten, dass die Willkürfreiheit der Beteiligten an Tauschakten sich zu willkürlicher Beherrschung verdichtet, wo grundlegende Asymmetrien (z. B. am Arbeitsmarkt) die Transaktionen bestimmen. Dann wäre aber ein Großteil einer liberalen Privatrechtsordnung, die Vertragsfreiheit gewährt, nach den Definitionen von Lovett unter nicht-idealen Bedingungen ungleicher Ressourcenausstattung, asymmetrischer Beziehungen und auch unvollkommener Märkte als Fall willkürlicher Beherrschung anzusehen, eine Konsequenz, die Frank Lovett jedoch nicht zieht, die sich aber mit seiner prozeduralen Definition von „arbitrariness“ bestens verträgt. In Präzisierung der Pettitschen Überlegungen formuliert Lovett: "More precisely, let us define social power as arbitrary to the extent that its potential exercise is not externally constrained by effective rules, procedures, or goals that are common knowledge to all persons or groups concerned" (Lovett 2010, S. 96). Arbitrariness ist daher weder Ausdruck, dass etwas zufällig oder unvorhersehbar geschieht (random and unpredictable) noch eines Ermessensspielraums (discretion). Ermessen kann durchaus nicht-willkürlich sein, wenn es eine Form der Delegation von klar umrissenen Entscheidungsspielräumen innerhalb klar definierter (rechtlicher) Regeln darstellt. Arbitrariness ist auch nicht abhängig von den Einstellungen jener Personen, die Herrschaft ausüben: Selbst eine wohlwollende, auf Fairness und Toleranz ausgerichtete Herrschaft ist arbiträr, wenn sie nicht personen-extern durch effektive, sanktionsbewehrte Regeln eingeschränkt ist.

15 Ein zentrales Problem tritt bei dieser scheinbar rein empiriebezogenen Definition auf, die aber als Basis einer Gerechtigkeitstheorie, die jede Form von Beherrschung als ungerecht ansieht, zugleich normativen Charakter besitzt: Die Theorie muss als utilitaristische Theorie messen, in welchem Maße durch welche konkreten Aktivitäten an welcher Stelle Beherrschung verringert worden ist, sie muss sogar in der Lage sein, das Anwachsen von Beherrschung auf einem Felde mit der Verringerung von Beherrschung in einem anderen Felde zu verrechnen, ein Problem, das auch dann nicht trivial ist, wenn man von dem gleichen Wert aller Menschen ausgeht, also nicht präferenzintensivere Personen bei Verbesserung ihrer Lage höher bewertet als präferenzschwächere Personen. Und sie muss den Wert der Gerechtigkeit gegen andere Werte anderer Theorien abwägen (Lovett 2010, S. 188). 
"Arbitrariness, so defined, arises when there are gaps in the network of effective social conventions (social norms, coordination conventions, laws, etc.) governing the possible exercise of social power. Sometimes these gaps are accidental or unintended, and sometimes they exist merely because appropriate social conventions have not yet been introduced. Other times, however, these gaps are explicitly created and sheltered by the surrounding configuration of social conventions. An example of the latter is traditional family law, which was specifically designed to prevent external interference with the authority of husbands and parents" (Lovett 2010, S. 111).

Nicht zufällig ist das Familienrecht aber Teil des Privatrechts und steht damit in enger Verbindung mit dem Vertragsrecht. Unter nicht-idealen Umständen ungleicher Ressourcenverteilung von Vermögen und Einkommen und in Anbetracht nicht-vollkommener Märkte existieren aber, durch das Vertragsrecht ermöglicht, Spielräume für vertragliche Beherrschung in einem strikten Sinne. Gerade dort, wo die Privatrechtsordnung ohne intensivere soziale Regulierung und Eingriffe in die Vertragsfreiheit Bestand hat, genau dort wird der Willkürfreiheit im Sinne Lovetts Raum gelassen. Privatrecht ist gerade die Sicherung eines Freiraums innerhalb des Netzwerkes an sozialer und rechtlicher Regulierung, es ist die Schaffung eines Raums eigenverantwortlicher Entscheidung ohne Bindung an andere Normen als jene, die die Freiheit als Willkürfreiheit für alle garantieren. Das moderne Recht ist im Unterschied zur Moral gerade auf die Entstehung solcher Räume angelegt, ${ }^{16}$ damit aber auch auf die Entfaltung von Räumen der Lovettschen Arbitrariness ausgerichtet! Sicherlich ist die Entwicklung des modernen Rechts von der regulatorischen Überformung des Vertragsrechts, des Haftungsrechts, des Familienrechts mit den Erweiterungen hin z. B. zum Arbeitsrecht und zum Verbraucherrecht bestimmt, doch ist der auf Willkürfreiheit zielende Kern der privaten Autonomie dadurch nicht eliminiert, nur eingeengt und spezifiziert worden. $\mathrm{Zu}$ unterstellen, dass diese Willkür dort, wo sie im Rahmen von Gesetzen zur Entfaltung kommen kann, keine Asymmetrien erzeugen würde, verkennt grundlegend die Verhandlungssituationen auf Märkten, die - unter der Annahme, dass ein Akteur ,im Spiel' bleiben will - nicht die Wahl lassen, sich auf beherrschende Vertragskonstruktionen mit hoher einseitiger Abhängigkeit einzulassen oder nicht. Dass Marktprozesse zu einem Prozess der Akkumulation von Ungleichheit führen, legt Lovett selbst ausführlich sogar für den perfekten Markt dar:

"Naturally, there will be winners and losers. Some people will make bad choices such as investing in a business that fails, or choosing a career in an industry that moves overseas; and some will suffer bad luck - such as developing a debilitating medical condition, or losing their home in a tornado. Conversely, others will make good choices or enjoy good luck - they will invent an incredibly popular new product, or happen to be born with highly valued natural talents. Thus, even if we start

16 Man vergleiche dazu Jürgen Habermas: „Während uns die Moral Pflichten auferlegt, die alle Handlungsbereiche lückenlos durchdringen, schafft das moderne Recht Freiräume für private Willkür und individuelle Lebensgestaltung. Unter der revolutionären Prämisse, dass rechtlich alles erlaubt ist, was nicht explizit verboten wird, bilden nicht Pflichten, sondern subjektive Rechte den Anfang für die Konstruktion von Rechtssystemen. Für Hobbes und das moderne Recht ist maßgebend die gleichmäßige Ermächtigung aller Personen, im Rahmen der Gesetze tun und lassen zu dürfen, was ihnen beliebt“" (Habermas 2011, S. 24). 
out with equal shares of goods, socioeconomic inequalities will inevitably arise. Moreover, these inequalities will continue to accumulate, both over the course of individual lives and, more significantly, from one generation to the next" (Lovett 2010, S. 195).

Diese Asymmetrieerzeugung eingestanden folgt aus der Definition von Beherrschung, dass zwischen dem Privatrecht und Beherrschung eine enge Beziehung besteht. Die Theorie der Beherrschung kommt wider Willen zu dem Ergebnis, dass der Kern des bürgerlichen Privatrechts Grundlage vielfältiger Beherrschung ist. Beherrschungsminimierung verlangte mithin, den Willkürspielraum, den das Privatrecht bietet, deutlich einzuschränken. Eine gegen Beherrschung gerichtete Gerechtigkeitstheorie müsste sich danach der regulativen Durchdringung des Privatrechts mit dem Ziel der Minimierung der Willkürfreiheit widmen.

\section{Eine gerechte Gesellschaft jenseits des Wohlfahrtsstaates: Die Property- Owning Democracy}

Diese Überlegungen zu Möglichkeiten der gerechtigkeitstheoretischen Erfassung von Transaktionen vergrößern zunächst die Zweifel daran, institutionelle Designs für eine Ökonomie entwickeln zu können, die im strengen Sinne den Anforderungen einer Gerechtigkeitstheorie, die sich den Sinn für deutlich ungleiche Verteilungsverhältnisse als kritisierenswerte Tatbestände bewahrt hat, genügen könnte. Rawls selber hat aber mit der "property-owning democracy“ eine Skizze einer solchen Verfassung der Ökonomie vorgelegt, die über die kapitalistische Marktökonomie hinausweist. John Rawls verfügt aufgrund der Anlage seiner Gerechtigkeitstheorie und Theorie des politischen Liberalismus als Idealtheorie über keine Konzepte für Übergänge, Transitionen, gewaltlose Brüche oder gar Strategien der Transformation. Der Weg, wie eine wohlgeordnete Gesellschaft erreicht werden könnte, bleibt daher notwendig ungeklärt. Das konnte solange auch als wenig bedeutsam erscheinen, als seine Theorie weithin als ein Entwurf galt, der eine ausgebaute Wohlfahrtsstaatlichkeit unterstützt. Ein weiteres Fortschreiten auf dem Weg der menschenrechtlichen Weiterungen und sozialstaatlichen Reformen schien das praktische Pendant zum theoretischen Entwurf Rawls' zu sein. Erst in "Justice as Fairness" aus dem Jahre 2001 sind Passagen enthalten, die der unterstellten Allianz zwischen Rawlscher Theorie und wohlfahrtsstaatlicher Reformpraxis widersprechen (Rawls 2003, S. 29, 211-218) und eine „property-owning democracy“ (und auch einen „liberal socialism“) dem „welfare state capitalism“ eindeutig vorziehen. Rawls selbst weist darauf hin (2003, S. 90 Fn. 17 und S. 211 Fn. 2), dass bereits in „A Theory of Justice“ die ordnungspolitischen Alternativen samt der Demokratie mit Eigentumsbesitz ${ }^{17}$ erörtert worden seien, allerdings

17 Den Begriff übernimmt Rawls vom Ökonomen und Nobelpreisträger des Jahres 1977 James Edward Meade. Zur Herkunft und Geschichte dieses Begriffs vgl. Jackson 2012. 
ohne die Unterschiede zum kapitalistischen Wohlfahrtsstaat ${ }^{18}$ deutlicher herauszuarbeiten (sehr früh dazu: Krouse u. McPherson 1988). Allerdings scheint dies eher eine Frage der Rawls-Rezeption denn des Textes der „Theorie der Gerechtigkeit“ zu sein, denn dieser beschreibt klar (Rawls 1975, S. 308-318) die Vorzüge eines marktsozialistischen Modells mit dezentralisiertem Privateigentum, auch wenn das Kapitel über die Verteilung mit der Bemerkung beginnt, die Frage von Privateigentum oder Sozialismus im Rahmen der Theorie als offen anzusehen (Rawls 1975, S. 291). In „Justice as Fairness“ akzentuiert Rawls seine Sicht der wirtschaftspolitischen Ordnungsmodelle durch die Gegenüberstellung von fünf Konzeptionen, die er danach gewichtet, ob sie als Realisierung der zwei Gerechtigkeitsprinzipien der Theorie der Gerechtigkeit gewertet werden könnten. Dass ein Laissez-faire-Kapitalismus und ein Staatssozialismus ${ }^{19}$ nicht den Gerechtigkeitsprinzipien genügen, ist keiner weiteren Erörterung bedürftig. Dass aber nicht der wohlfahrtsstaatliche Kapitalismus, sondern nur ein liberaler Sozialismus oder die „Demokratie mit Eigentumsbesitz“ als angemessene Konzepte der Umsetzung der Gerechtigkeitsprinzipien erscheinen, mag vor dem Hintergrund der Rezeptionsgeschichte überraschen. Rawls ist danach eher als ein moderater Marktsozialist denn als Vertreter des Sozialstaatsmodells einzustufen, dem er vorwirft Vermögenskonzentrationen zuzulassen, die die Steuerung der Politik durch kleine Gruppen der Gesellschaft nach sich ziehen und die Gleichheit der Bürger als Bürger zerstören. Er verteidigt sowohl das Recht auf persönliches Privateigentum (Rawls 2003, S. 180, 216) als auch die Verteilung der Güter über Märkte (Rawls 1975, S. 298-308), kombiniert dies allerdings mit Institutionen zur Neutralisierung und Dezentralisierung des Produktivmittelbesitzes.

Derartige Modelldiskussionen und -konstruktionen, wie sie Rawls anstellt, finden sich nur vereinzelt in der Literatur. Dies hat der verdienstvolle Sammelband von O'Neill und Williamson zur Property-Owning Democracy (2012) deutlich gemacht, der neben Roemer (1994) vor allem noch auf Alperovitz (2011) und Schweickart (1993) verweist. Der inzwischen nicht behobene Mangel an ausgearbeiteten Entwürfen zeigt sich auch an der Knappheit der Rawlsschen Skizzen. Die beiden präferierten Modelle des liberalen Sozialismus und der Demokratie mit Eigentumsbesitz unterscheiden sich nur geringfügig (O’Neill 2012, S. 76), vor allem in der Besitzstruktur. Im Modell des liberalen Sozialismus (demokratisches politisches Regime, freie Wettbewerbsmärkte, Unternehmen in Arbeiterhand und annähernd gleiche Verteilung von Produktionsmitteleigentum und natürlichen Ressourcen, so die vierteilige Bestimmung in Rawls 2007, S. 323) ist das Eigentum an Produktionsmitteln vergesellschaftet (Rawls 2003, S. 215-216) bei einer

18 Rawls spricht (2001, S. 137-140) von „,welfare-state capitalism“, während die deutsche Übersetzung Attribution und Nomination wechselt und von einem „kapitalistische[n] Wohlfahrtsstaat“ spricht (2003). Die Originalfassung macht in der Aufzählung aller fünf Ordnungsmodelle deutlicher, dass der Gegensatz capitalism-socialism die dominante Unterscheidung ist (Laissez-faire capitalism, welfare-state capitalism, state socialism, liberal [democratic] socialism). Allein die Property-Owning Democracy als das von Rawls bevorzugte Modell entkommt terminologisch diesem Grundschema. In „Justice as Fairness“ wird klargestellt, dass die Property-Owning Democracy eine Alternative zum Kapitalismus darstellen soll (Rawls 2003, S. 211).

19 Die Marxsche Vision des vollendeten Kommunismus gilt Rawls als Konzeption einer Gesellschaft jenseits der Gerechtigkeit (Rawls 2007, S. 322, Rawls 2003, S. 272). 
Vielfalt von selbstverwalteten Firmen, die auf freien Märkten gegeneinander konkurrieren. Die Demokratie mit Eigentumsbesitz erlaubt dagegen auch Privatbesitz an Produktivvermögen, aber nicht als Grundrecht, sondern als wirksamstes Mittel, um das Differenzprinzip zu realisieren (Rawls 2003, S. 271). Eine gerechte Grundstruktur einer Gesellschaft, so Rawls, kann nicht auf Einkommensumverteilungen und Sicherungen gegen soziale Risiken allein basieren, sie kann nur gelingen, wenn das Eigentum an Produktionsmitteln nicht konzentriert oder gar monopolisiert ist. Eine gerechte Gesellschaft muss, wenn sie das Eigentum an Produktionsmitteln nicht vergesellschaften will, einen institutionellen Mechanismus etablieren, der die durch Marktprozesse und bei Privateigentum immer neu entstehenden Vermögensungleichheiten genau dann reduziert, wenn es zu einem Aus$\mathrm{maß}$ an Konzentration kommt, das die politische Selbstbestimmungsfähigkeit der Bürger zu beeinträchtigen droht. Eine gerechte Gesellschaft benötigt eine Strategie der Vermögensdezentralisierung, sie bedarf der Instrumente und Institutionen, die dafür sorgen, dass Vermögenskonzentrationen abgebaut werden können, und dies nicht nur einmalig, sondern als wiederkehrender Vorgang, immer unterstellt, dass sich neuauftretende Einkommens- und Vermögensungleichheiten nicht verhindern lassen. ${ }^{20}$ Rawls kombiniert eine marktlich verfasste Ökonomie mit einer dezentralisierten Verteilung der relevanten Vermögen, also insbesondere dem Eigentum an Produktionsmitteln, bietet als Maßnahmen aber nur eine progressive Erbschaftsteuer und eine proportionale Ausgabensteuer an, die sogar an die Stelle einer progressiven Einkommensteuer treten könnte; direkte Eingriffe in das Transaktionsgeschehen gelten als nicht erforderlich (Rawls 2003, S. 248-249). Wie eine progressive Erbschaftsteuer und Ausgabensteuer eine Vermögenskonzentration verhindern sollen, wird jedoch nicht gezeigt. Sie sind sicherlich in der Lage, Konzentrationsprozesse zu verlangsamen, eine Umverteilung führen sie aber nicht herbei. Zudem fehlt jegliches Maßnahmenkonzept als Teil der Grundstruktur, das Möglichkeiten bieten würde, einmal eingetretene Vermögenskonzentrationen (z. B. durch nicht angepasste, d. h. zu niedrige Steuersätze) wieder rückgängig zu machen. Auch für den liberalen Sozialismus ist nur geklärt, dass es qua öffentlichen Eigentums keine Vermögenskonzentration geben kann. Wie aber wird die durch Marktprozesse immer mögliche ungleiche Entwicklung der von gewählten Managern geleiteten oder von Arbeitern selbstverwalteten Unternehmen (Rawls 2003, S. 273) aufgefangen und wie kann die Konzentration wirtschaftlicher Verfügungsmacht bei wenigen Unternehmen verhindert werden?

Angesichts des doch recht begrenzten Maßnahmenbündels, das Rawls der Demokratie mit Eigentumsbesitz als Teil der Grundstruktur zuweist, ist zu fragen, worin das Spezifikum der Demokratie mit Eigentumsbesitz besteht (auch wenn in Rechnung gestellt wird, dass eine breite Streuung des Bildungskapitals zum Konzept der Dekonzentration der ,Vermögen“ und ,Produktivkräfte‘ gehört, Rawls

20 O’Neill zählt nur die Verhinderung intergenerationeller Vorteilsübertragung zu den Defintionsmerkmalen der Property-Owning Democracy („1. Wide dispersal of capital“, „2. Blocking the intergenerational transmission of advantage“, „3. Safeguards against the ,corruption“ of democratic politics “; O’Neill 2012, S. 80-81). Aber auch gegen intragenerationelle Konzentrationsbewegungen größeren Ausmaßes müssen zur Einhaltung des Rawlsschen Konzepts Vorkehrungen getroffen werden können. 
2003, S. 218). Rawls charakterisiert den Unterschied zwischen einer PropertyOwning Democracy und dem Welfare-State Capitalism wie folgt:

\begin{abstract}
"One major difference is this: the background institutions of property-owning democracy work to disperse the ownership of wealth and capital, and thus to prevent a small part of society from controlling the economy, and indirectly, political life as well. By contrast, welfare-state capitalism permits a small class to have a near monopoly of the means of production. Property-owning democracy avoids this, not by the redistribution of income to those with less at the end of each period, so to speak, but rather by ensuring the widespread ownership of productive assets and human capital (that is, education and trained skills) at the beginning of each period, all this against a background of fair equality of opportunity" (Rawls 2001, S. 139).
\end{abstract}

Die Idee der Periodenbetrachtung ist erhellend, auch wenn sie von Rawls nur aus Darstellungsgründen eingeführt wird und eher metaphorisch anzusehen ist (O'Neill 2012, S. 90). Wenn die Nebenfolgenkumulation größere Ungleichheiten im Vermögens- bzw. Produktionsmittelbesitz erzeugt, bedarf es periodisch einer Korrektur, um wieder einen weitgestreuten Besitz herzustellen. Während Einkommen im Wohlfahrtsstaat nachträglich umverteilt werden, sind Eigentum und Humankapital in der Demokratie mit Eigentumsbesitz vorgängig dekonzentriert. Diese Differenzierung zwischen Einkommensverteilung und Vermögensverteilung wird in einer Mehrperiodenbetrachtung jedoch weniger bedeutend, muss es doch darauf ankommen, Akkumulationen gleich welcher Art, im Einkommens- wie im Vermögenssektor, schon aufgrund der wechselseitigen Beziehungen und ihrer Transferierbarkeit zu verhindern. Ob Einkommens- oder Vermögensverteilung, die ökonomischen Konzentrationsbewegungen müssen ständig oder in periodischen Abständen korrigiert, die Reichtümer abgeschöpft werden, um mit einem egalitäreren Ausgangszustand in die nächste Runde der Transaktionen unter Bedingungen der fairen Chancengleichheit zu starten. Während hier auf die Schwierigkeiten hingewiesen werden soll, die sich einer Dekonzentrationspolitik gleich welcher Art stellen, hält O'Neill (2012) bereits ein eher sozialstaatliches Modell für ausreichend, um die Anforderungen vor allem des ersten Gerechtigkeitsprinzips zu genügen.

\title{
5. Moralisierung der Ökonomie?
}

Selbst mitten in der Finanz- und Eurokrise finden Überlegungen zu einem neuen Verhältnis von Politik und Ökonomie, zu einer, gerechteren Ökonomie' oder ihrer Demokratisierung in der öffentlichen Debatte praktisch keine Resonanz. Die Forschungen zu den in den Massenmedien vorherrschenden Legitimationsmus- 
tern ${ }^{21}$ vermögen dies zu belegen. Eine durchaus weit verbreitete Kritik des Marktes und der Marktwirtschaft bündelt sich weder zu marxistischen, anarchistischen, genossenschaftlichen noch erneuerten keynesianischen (dazu: Skidelsky 2009) Mustern. Auch die Umkehrung schumpeterianischer, neoklassischer, hayekianischer, ordoliberaler oder evolutionsökonomischer Begründungsmuster der Marktwirtschaft findet nur in Ansätzen statt. Zwar sind in der Krise die Zweifel an der Glaubwürdigkeit, Effizienz und Effektivität dieser Ordnung gewachsen, aber die Kritik der Funktionsfähigkeit dominiert nicht die Debatten. Argumentationen, die die mangelnde Berücksichtigung der Interessen der Bevölkerung oder fehlende demokratische Verantwortung der ökonomischen Akteure beklagen, finden auch in der Krisenzeit nach 2008 in drei von vier Ländern (Deutschland, Großbritannien und USA) sogar weniger Zustimmung. ${ }^{22}$ Die Demokratisierung der Ökonomie ist gerade in der Krise kein Thema. Auch Gerechtigkeitsargumente rücken durch die Krise nicht in den Vordergrund. ${ }^{23}$ Noch am ehesten lässt sich eine moralisch-ethische Kritik der Marktwirtschaft als in der Krise bedeutender werdendes Cluster von Argumenten identifizieren. ${ }^{24}$ Drei einzelne Argumente sind hier tragend. Erstens: Die Marktordnung zerstöre die moralischen Grundlagen der Gesellschaft, sie korrumpiere die Menschen und mache sozialen Zusammenhalt und Solidarität unmöglich. Zweitens: Cliquenwirtschaft und mangelnde Führungstugenden kennzeichneten die gegenwärtige Gestalt des Kapitalismus. Drittens: Es sei die pure Gier, die im „Turbokapitalismus“ regiere, die Tugenden des ehrbaren Kaufmanns seien dem weit über jede vernünftige Strategie der Einkommenserzielung hinausgehenden Streben nach Geld um des Geldes willen gewichen. Gerade das letzte Argument bezieht sich vor allem auf die Träger der Marktwirtschaft: die Manager, Aktionäre, „Finanzjongleure“, Unternehmer und Banker. In der an politischen Ordnungen entwickelten Legitimationstheorie gilt aber eine Kritik, die nicht die Ordnung als ganze, sondern nur die Träger dieser

21 Die Ausführungen zur empirischen Erforschung der Legitimation von Marktökonomien beruhen auf den Arbeiten des Teilprojektes B1 im Rahmen des Bremer DFG-Sonderforschungsbereichs „Staatlichkeit im Wandel“, die im Team mit Dominika Biegon, Jennifer Gronau, Sebastian Haunss, Falk Lenke, Henning Schmidtke und Steffen Schneider erstellt worden sind. Die für politische Ordnungen erprobten Verfahren der Kommunikationsforschung mit der Untersuchung von Legitimationsstatements in Qualitätszeitungen werden in abgewandelter Form genutzt, um die öffentliche Rechtfertigung und Kritik wirtschaftlicher Ordnungen (Marktwirtschaft/Kapitalismus) und deren wesentlicher Grundprinzipien (freier Markt, Wettbewerb, Privateigentum) zu erkunden. Für einen Zeitraum von 14 Jahren (1998 bis 2011) wurden für vier Länder (USA, Großbritannien, Schweiz und Deutschland) in je zwei Qualitätszeitungen unterschiedlicher politischer Couleur bewertende Aussagen zur Marktwirtschaft und ihren Grundprinzipien identifiziert.

22 Demokratische Legitimationsmuster im Vergleich der Jahre 1998-2007 als Vorkrisenjahren zu den Krisenjahren 2008-2011: Schweiz Anstieg von 7,7\% auf 9,9\% aller Legitimationsstatements, Deutschland Rückgang von $10,3 \%$ auf $6,3 \%$, Großbritannien von $12,7 \%$ auf $7,9 \%$ und USA von $11,1 \%$ auf $8,8 \%$.

23 Gerechtigkeitsbezogene Legitimationsmuster im Vergleich der Jahre 1998-2007 als Vorkrisenjahren zu den Krisenjahren 2008-2011: Schweiz leichter Rückgang von 8,4\% auf 8,1\% aller Legitimationsstatements, Deutschland stärkerer Rückgang von 9,6\% auf 6,9\%, Großbritannien leichter Anstieg von $11,6 \%$ auf $12,0 \%$ und USA mit einem Rückgang von $14,4 \%$ auf $11,8 \%$.

24 Moralische Legitimationsmuster im Vergleich der Jahre 1998-2007 als Vorkrisenjahren zu den Krisenjahren 2008-2011: Schweiz Anstieg von 14,8\% auf 18,0\% aller Legitimationsstatements, Deutschland von $13,6 \%$ auf $15,8 \%$, Großbritannien von $8,5 \%$ auf $13,0 \%$ und USA von $5,6 \%$ auf $18,6 \%$. 
Ordnung als Kollektiv angreift, als vergleichsweise am geringsten weitreichend (Norris 2011). So lässt sich durchaus ein Aufstieg eines Moral-Frames in der Finanz- und Schuldenkrise aufzeigen, mit der Anklage der Gier der Banker/Manager als Hauptargument. Zugleich sind derartige Anklagen selten mit Reformkonzepten oder alternativen Modellen ökonomischer Ordnung verbunden. ${ }^{25}$

Den Aufstieg moralbezogener Argumentationsmuster reflektiert auch die wirtschaftsethische Literatur. Die Forderung nach Moralisierung der Unternehmenspolitik nutzt die Impulse der Corporate-Social-Responsibility-Diskussion zum philosophischen Ausbau einer Unternehmensethik (z. B. Neuhäuser 2011), verbleibt dabei aber im Rahmen einer marktwirtschaftlichen Logik. Derartige Unternehmensethiken werden aktuell durch eine Philosophie der Grenzen des Marktes überboten, die normative Maßstäbe entwickelt, um bestimmte Güter oder Vorgänge entsprechend den (verheerenden) Folgen für die Beteiligten vor, während und nach dem Tauschprozess der Vermarktlichung zu entziehen (Satz 2010; Sandel 2012). Noch weitergehender sind Beiträge in der Nachfolge gerechtigkeitstheoretischer Debatten, die eine Moralisierung nicht nur der Unternehmen oder einzelner Sphären, sondern der Ökonomie insgesamt einfordern.

Der Bezug zu Moralkategorien jenseits der Gerechtigkeit kennzeichnet gerade einen der wenigen Versuche, über Alternativmodelle zur kapitalistischen Marktökonomie im Rahmen der politischen und Sozialphilosophie nachzudenken. Gerald A. Cohen hat kurz vor seinem Tod einen kleinen Band „Why not Socialism?“ veröffentlicht, der Grundprinzipien einer sozialistischen Gesellschaft zu erläutern versucht. In „Rescuing Justice and Equality“ (2008, insb. S. 16-17, 116-150) hatte Cohen bereits seine Kritik des Rawlsschen Differenzprinzips als Maßstab der Verteilungsgerechtigkeit mit seiner Konzentration auf die Grundstruktur vorgetragen (differenzierend: Ronzoni 2007, 2008). Cohen versteht die Grundstruktur als Gesamtheit der auf öffentlichen Zwangsgesetzen beruhenden Institutionen. Während die Bürger in den Institutionen auf Gleichheit und Gerechtigkeit hinarbeiten, bliebe bei Rawls, so Cohens Argumentation, das individuelle Verhalten der Bürger in ökonomischen Kontexten unreguliert und werde von Nutzenmaximierungsüberlegungen geprägt. Dies erscheint Cohen als „double-minded“-Modell (Cohen 2008, S. 123), das keine geeignete Grundlage für eine gerechte Gesellschaft abgeben könne:

"A society that is just within the terms of the difference principle, so we may conclude, requires not simply just coercive rules, but also an ethos of justice that informs individual choices. In the absence of such an ethos, inequalities will obtain that are not necessary to enhance the condition of the worst off: the required ethos promotes a distribution more just than what the rules of the economic game by themselves can secure. And what is required is indeed an ethos, a structure of response lodged in the motivations that inform everyday life [...]" (Cohen 2008, S. 123).

25 Die kapitalismuskritische Literatur konzentriert sich meist auf die Dechiffrierung der aktuellen Lage, auf die Anklage eines derartigen Systems und die Aufforderung zur moralisch-affektuellen Gegenwehr (paradigmatisch: Hessel 2011; andere Beispiele: Schirrmacher u. Strobl 2010, Schulze 2012, Wingert 2013). 
Diese Einforderung eines Ethos als zwingend erforderlicher Grundlage einer gerechten Gesellschaft setzt sich in Cohens Sozialismusmodell fort.

Dass Cohen als Paradigma seines Sozialismusbildes einen „camping trip“, ein Zeltlager (so die deutsche Übersetzung, Cohen 2010) wählt, ist ein Anzeichen dafür, in welchem Maße sich Cohen von der Vorstellung der Gemeinschaftlichkeit leiten lässt. Miriam Ronzoni (2012) hat bereits gezeigt, dass bestimmte Eigenschaften des Zeltlagers selbst auf andere kleinteilige, aber grundlegende gesellschaftliche Nahbeziehungen wie die Familie nicht übertragbar sind. Problematisch ist sicherlich auch, dass die gruppeninternen Interaktionen, z. B. die nötigen Einigungen darüber, was wann von wem und mit wem auf dem Campingtrip getan wird, nicht näher betrachtet werden. Wichtig ist Cohen, dass es innerhalb der Gruppe einen Ausschluss des Markttausches via Preisen und das Bemühen um eine gemeinsame Freizeitgestaltung gibt. Gleichheit und Gemeinschaft sind die Prinzipien, die den Sozialismus bestimmen. Gleichheit wird als radikale Chancengleichheit verstanden, die aber mit Ergebnis-Ungleichheiten einhergeht, die aus differierenden Präferenzen und Glück folgt, das bei Beteiligung an Marktprozessen unvermeidlich wird. Diesen Ungleichheiten muss durch das Prinzip gemeinschaftlicher Gegenseitigkeit entgegengearbeitet werden. Das friktionslose Funktionieren der Interaktion zwischen den Campern gründet sich neben der Gleichheit daher vor allem auf dem Prinzip der Gemeinschaft, die negativ durch Ausschluss von egoistischen Motiven, Angst und Gier, positiv durch die Orientierung an Kooperation als Ziel an sich bestimmt ist: „Bei gemeinschaftlicher Gegenseitigkeit ist unser Verhältnis zueinander nicht - wie im Marktkontext - nur ein instrumentelles, in dem ich gebe, weil ich etwas dafür bekomme. Unser Verhältnis ist stattdessen ein nicht-instrumentelles. Ich gebe, weil mein Gegenüber etwas braucht oder will, und ich erwarte von ihm vergleichbare Großzügigkeit“ (Cohen 2010, S. 44). Als Antwort auf die Frage, wie ein ökonomisches System oder eine institutionelle Makrostruktur aussehen könnte, die diesen Ansprüchen an Gemeinschaftlichkeit, Gleichheit und Großzügigkeit näher kommen könnte, verweist Cohen auf marktsozialistische Modelle, insbesondere das von John E. Roemer, erkennt aber deutlich dessen doch nur sehr begrenzte Bekämpfung der egoistischen Motive. Cohens Ausführungen enden daher mit der radikalen Kritik des Marktprinzips: „Jeder Markt, sogar ein sozialistischer Markt, ist ein räuberisches System“ (Cohen 2010, S. 77). Nur eine Gesellschaft, die grundlegend vom Prinzip und Ethos gemeinschaftlicher Gegenseitigkeit getragen ist, kann einen Ausweg bieten. ${ }^{26}$

In der Theoriedebatte finden sich auch Gegenstrategien einer moralisierenden Bejahung des Kapitalismus. Die Marktwirtschaft erscheint dann als moralisch gebotene Form der gesellschaftlichen Organisation der Ökonomie. Die alten Effizienz- und Freiheitsargumente rücken an zweite Stelle oder werden im Sinne einer Moraltheorie reinterpretiert. Als Beispiel sei hier wieder Tomasi (2012) herange-

26 Es wäre zu prüfen, ob die aktuell prominenteste Stimme der Occupy-Bewegung, David Graeber, in seinen anthropologisch-ökonomischen Schriften (2001, 2012a) mit dem Rückgriff auf Marx und Mauss bzw. in seinen politischen Schriften (2012b, 2013) mit anarchistischen Vorstellungen und Hinweisen auf Kropotkins Gegenseitigkeit über das Konzept von Cohen hinauskommt. 
zogen. ${ }^{27}$ Er rechtfertigt die „Marktdemokratie“ mit ihrem moralischen Wert auch für die Schlechtestgestellten. Es ist der moralische Wert der Selbstautorenschaft, der gegen staatliche Eingriffe und Transfers angeführt wird. Nur, was selbst erarbeitet und als eigener Verdienst angesehen werden kann, sichert eine auf Selbstautorenschaft beruhende Selbstachtung. Mit Selbstautorenschaft ist hier eine positiv konnotierte Formel an die Stelle der immer mit externer Zuschreibung, einem gewissen Maß an Zumutung und vor allem Verpflichtung einhergehenden Formel der Eigenverantwortung getreten, die zudem den Vorteil bietet, die Assoziation von Autonomie und Selbstbestimmung zu wecken, aber im Sinne einer rein marktbezogenen Leistungsgerechtigkeit zu interpretieren. Leistungsgerechtigkeit und Verdienst werden hier an den Markterfolg eng gebunden. Als „moral value“ gilt es, sich den Marktbedingungen und den dort herrschenden vorliegenden Möglichkeiten zu überlassen. Leistungsgerechtigkeit kann dagegen auch ein kritischer Begriff gegenüber einem puren Marktlauf werden, wenn man fragt, ob die Vorstandsvorsitzenden gewährten Antrittsprämien, Gehälter, Boni, Ruhestandszusagen, Abfindungen etc. in Millionenhöhe wirklich aufgrund ihrer persönlichen Leistung und Anstrengung verdient sein können. Dann ist der Begriff der Leistung noch von dem des Markterfolges geschieden, was Hayek (2003) in durchaus markt-affirmativer Perspektive dazu gebracht hat, dem Markt die Fähigkeit zur Schaffung von Leistungsgerechtigkeit abzusprechen - und nicht nur die zur Schaffung sozialer Gerechtigkeit. Bei Tomasi und anderen Beiträgen zur aktuellen Gerechtigkeitsdiskussion (zur Kritik: Nullmeier 2010, grundlegend: Neckel 2008) wird dagegen Markterfolg und Leistung in eins gesetzt. Die am Markt erzielbaren Einkommen sind per se leistungsgerechte Einkommen, jede Distanz zum Marktgeschehen und damit ein Ruf nach Korrekturen durch kollektive Organisation oder politische Intervention verbietet sich. Streecks (2013, S. 235) Schreckensbild des hinter der Vorstellung von purer Marktgerechtigkeit vereinigten Volkes wird hier als normatives Ideal - und zwar in der Form einer Gerechtigkeits- und Moraltheorie - propagiert. Eine legitimatorische Restabilisierung dürfte wohl am ehesten auf diesem Wege gelingen: Alle Entwicklungen des Marktes müssen als Ausdruck von Markt- und Leistungsgerechtigkeit erscheinen und als moralisch wertvoll geadelt werden. Es ist ein moraltheoretisch interpretierter Marktfatalismus, der als höchstes Ideal einer normativen Rechtfertigung des Marktes erscheinen muss. Aber auch Tomasi vermag dies so lange nicht zu gelingen, wie mit dem Begriff der Selbstautorenschaft ein Kriterium gegeben ist, das doch noch kritisch gegen Marktentwicklungen und den impliziten Selbstlauf derselben ohne Mitwirkungs- und Mitbestimmungsmöglichkeiten für den einzelnen gewendet werden kann.

27 Ein anderes Beispiel: Shiller (2012) kombiniert seinen Aufruf für eine ,Demokratisierung bzw. Expansion des Finanzsystems mit moralischen Anforderungen an die Akteure in den Finanzinstitutionen - eine Art ökonomischen Tugendkatalog. 


\section{Schluss}

Angesichts der auch in der Krise fortbestehenden, wenn nicht gar gesteigerten Macht der Finanzmarktakteure ist das Szenario einer Transformation der Demokratie in eine Postdemokratie oder verwandter Diagnosen wie der einer in den USA regierenden „Winner-Take-All-Politics“ (Hacker u. Pierson 2010) mehr als nur eine Warntafel, aufgestellt von Theoretikern, die einem Goldenen Zeitalter starker Gewerkschaften und starker Sozialdemokratie nachtrauern. Doch jenseits dieser Diagnose fehlt es in der Postdemokratie-Debatte an Impulsen für eine Neufassung des Demokratieprojekts, die auch die Verursacherseite der Krisen einbeziehen würde. Entsprechend sollte sich die normative politische Theorie der Ökonomie zuwenden.

Praktisch mag das Einfordern von keynesianischer Ausgabenpolitik, Bewahrung des Sozialstaates, verstärkter Banken- und Finanzmarktaufsicht, von erhöhten Einkommen-, Erbschafts- und Vermögenssteuern und anderen Maßnahmepaketen im Rahmen einer „Sozialen Demokratie“ (Meyer 2009, 2011) ein wichtiger Schritt, vielleicht der aktuell einzig gangbare Weg sein. Die Theoriebildung wird jedoch angesichts der bereits eingetretenen extremen sozialen Ungleichheiten weit stärker die Frage der Reduktion bereits vorhandener und weiter steigender Ungleichheit - und nicht nur deren Moderation - bearbeiten müssen. Angesichts der entstandenen sozialen Ungleichgewichte und Vermögensdifferenzen und unter der Annahme, dass die basale Ungleichheitsakkumulationsthese richtig ist, sind neben Institutionen und Instrumenten, die die Neuakkumulation von Ungleichheit bremsen, auch Konzeptionen erforderlich, die eine Deakkumulation von Ungleichheiten als politische Aufgabe annehmen. Bisher haben einschneidende militärische, soziale und/oder politische Brüche den Weg für größere Umverteilungsbewegungen - sowohl der ökonomischen Ressourcen als auch der politischen Rechte - gewiesen. Krieg, Bürgerkrieg, Hyperinflation und Wirtschaftszusammenbruch, Totalitarismus und der Zusammenbruch von Diktaturen, Revolutionen und ökologische Katastrophen sind einige jener gesellschaftlichen Einschnitte, die in Form eines „Bruchs“ oder „Einschnitts“ sich auch auf die Ungleichheitslage ausgewirkt haben. Diese historische Auskunft stellt für normative Theorie nur eine weitere Belastung dar: Die normativ geforderte Deakkumulation als Akt gegen die weitere Ungleichheitssteigerung scheint unter Normalbedingungen einer friedlichen demokratischen Entwicklung kaum denkbar. Die theoretische Aufgabe besteht darin, die Möglichkeiten der Beendigung radikaler Ungleichheit ohne die in der Regel katastrophischen Erscheinungen jener historisch bekannten Formen gesellschaftlichen „Bruchs“ zu erkunden. Gefordert ist die Entwicklung von Deakkumulations-, Terminierungs- und Unterbrechungsmodellen, Modellen, die den Selbstlauf einer Ökonomie sich steigernder Ungleichheit stoppen. Vor aller Instrumentendiskussion im Steuerrecht (Erbschaft, Einkommen, Vermögen, Transaktionen) oder auf den Feldern der Schuldenerlasse, Insolvenzregeln, Abwertungen, Währungsreformen, die mindestens Kompetenzen der Politischen Ökonomie, Finanzwissenschaft, Privatrechtswissenschaft und PolicyForschung verlangen, sollte es eine Aufgabe einer normativen politischen Theorie 
sein, Formen der Terminierung und Unterbrechung überhaupt als legitime politische Maßnahmen anzuerkennen.

Damit ist aber eher das Niveau der Radikalität zukünftiger Theoriearbeit markiert. Die Herausforderung auf theoretischer Ebene liegt gemäß der vorgetragenen These darin, zugeben zu müssen, dass Transaktionen intern nicht demokratisierbar und auch nicht einer gerechten Gestaltung zugänglich sind - und dennoch an Demokratie- und Gerechtigkeitstheorie als Ankern politischer Theoriebildung festzuhalten. Wenn die Alternative einer allein auf Transaktionsregeln basierten Gerechtigkeitstheorie (wie der Entitlement-Theorie Robert Nozicks) durch das Wissen um die unvermeidlich sich akkumulierenden Nebenfolgen von Transaktionen zudem versperrt ist, bliebe nur zuzugeben, dass Transaktionen außerhalb des Horizonts der normativen Theorie stehen. Dann würde sich die Theoriebildung - durchaus in der unmittelbaren Nachfolge von John Rawls - auf eine normative Theorie der Institutionen beschränken, die vielleicht erweitert werden könnte um eine Theorie demokratisch verfasster Wirtschaftsorganisationen. Die alltagsweltliche Kritik an Transaktionen, die sich ja durchaus auch der Sprache der Ungerechtigkeit bedient, erhielte aber kein Pendant auf dem Boden normativer Theorie: Allokative Fragen ständen außerhalb der sich selbst auf Institutionen und die grundlegende Frage politischer Macht (Forst 2011, insb. S. 29-52) beschränkenden Gerechtigkeitstheorie. ${ }^{28}$ Benötigt normative Theorie einen jeweils eigenen Ansatz, wenn sie sich auf Transaktionen, Transaktionsnetze und basale Kommunikationen richtet statt auf Organisationen und institutionelle Grundstrukturen? Oder ist eine Theorie denkbar, die in einem Zugriff normative Konzepte für Transaktionen, Organisationen und die institutionelle Grundstruktur liefert?

\section{Literatur}

\section{Literatur}

Alperovitz, Gar. 2011. America Beyond Capitalism. Reclaiming Our Wealth, Our Liberty, and Our Democracy. Second Edition. Takoma Park: Democracy Collaborative Press.

Anderson, Elizabeth. 2013. Book Review: Free Market Fairness. Political Theory 41:163166.

Arnoldi, Jakob. 2009. Alles Geld verdampft. Finanzkrise in der Weltrisikogesellschaft. Frankfurt a. M.: Suhrkamp.

Aspers, Patrick. 2011. Markets. Cambridge: Polity Press.

Beckert, Jens. 1997. Grenzen des Marktes. Die sozialen Grundlagen wirtschaftlicher Effizienz. Frankfurt/New York: Campus Verlag.

Beckert, Jens, und Christoph Deutschmann (Hrsg.). 2009. Wirtschaftssoziologie, KZfSS Sonderheft 49. Wiesbaden: VS Verlag für Sozialwissenschaften.

28 Diese politische Konzeption ergibt sich nicht zwingend aus der Kritik des Distributionsparadigmas in der Gerechtigkeitstheorie, diese kann vielmehr recht unterschiedliche Neukonzeptionen begünstigen, wie ein Vergleich von Young (1990), Forst (2011) und Schemmel (2012) zeigen könnte. 
Beckert, Jens, Rainer Diaz-Bone und Heiner Ganßmann (Hrsg.). 2007. Märkte als soziale Strukturen. Frankfurt/New York: Campus Verlag.

Beitz, Charles R. 1989. Political Equality. Princeton: Princeton University Press.

Bermeo, Nancy, und Jonas Pontusson (Hrsg.). 2012. Coping with Crisis. Government Reactions to the Great Recession. New York: Russell Sage Foundation.

Bevir, Mark, und Frank Trentmann (Hrsg.). 2004. Markets in Historical Contexts. Ideas and Politics in the Modern World. Cambridge: Cambridge University Press.

Bispinck, Reinhard, Thorsten Schulten und Peter Raane (Hrsg.). 2008. Wirtschaftsdemokratie und expansive Lohnpolitik. Zur Aktualität von Victor Agartz. Hamburg: VSA Verlag.

Bofinger, Peter. 2012. Zurück zur D-Mark? Deutschland braucht den Euro. München: Droemer.

Brock, Gillian. 2009. Global Justice. A Cosmopolitan Account. Oxford: Oxford University Press.

Cohen, Gerald A. 2008. Recuing Justice and Equality. Cambridge/London: Harvard University Press.

Cohen, Gerald A. 2010. Sozialismus - warum nicht? München: Knaus.

Crouch, Colin. 2008. Postdemokratie. Frankfurt a. M.: Suhrkamp.

Crouch, Colin. 2011. Das befremdliche Überleben des Neoliberalismus. Berlin: Suhrkamp.

Dahl, Robert A. 1985. A Preface to Economic Democracy. Berkeley/Los Angeles: University of California Press.

Dahl, Robert A. 1998. On Democracy. New Haven/London: Yale University Press.

Dahl, Robert A., und Charles E. Lindblom. 1953. Politics, Economics, and Welfare. Planning and Politico-Economic Systems Resolved into Basic Social Processes. New York: Harper \& Row.

Demirovic, Alex. 2007. Demokratie in der Wirtschaft. Positionen, Probleme, Perspektiven. Münster: Westfälisches Dampfboot.

Deutschmann, Christoph. 2012. Die Legitimität des Kapitalismus: eine wirtschaftssoziologische Sicht. In Der Aufstieg der Legitimitätspolitik. Rechtfertigung und Kritik politisch-ökonomischer Ordnungen, Leviathan-Sonderband 27, Hrsg. Anna Geis, Frank Nullmeier und Christopher Daase, 245-261. Baden-Baden: Nomos.

Dworkin, Ronald. 2000. Sovereign Virtue. The Theory and Practice of Equality. Cambridge/London: Belknap Press of Harvard University Press.

Dworkin, Ronald. 2011a. Justice for Hedgehogs. Cambridge/London: Belknap Press of Harvard University Press.

Dworkin, Ronald. 2011b. Was ist Gleichheit? Berlin: Suhrkamp.

Elster, Jon, und Karl Ove Moene (Hrsg.). 1989. Alternatives to Capitalism. Cambridge: Cambridge University Press.

Fligstein, Neil. 2001. The Architecture of Markets. An Economic Sociology of TwentyFirst-Century Capitalist Societies. Princeton/Oxford: Princeton University Press.

Forst, Rainer. 2011. Kritik der Rechtfertigungsverhältnisse. Perspektiven einer kritischen Theorie der Politik. Berlin: Suhrkamp.

Fox, Justin. 2009. The Myth of the Rational Market. A History of Risk, Reward, and Delusion on Wall Street. New York: HarperCollins.

Freeman, Samuel. 2007. Rawls. London/New York: Routledge. 
Frydman, Roman, Michael D. Goldberg. 2011. Beyond Mechanical Markets. Asset Price Swings, Risk, and the Role of the State. Princeton/Oxford: Princeton University Press.

Graeber, David. 2001. Toward an Anthropological Theory of Value. The False Coin of Our Own Dreams. New York, Basingstoke: Palgrave.

Graeber, David. 2012a. Schulden. Die ersten 5000 Jahre. Stuttgart: Klett-Cotta.

Graeber, David. 2012b. Kampf dem Kamikaze-Kapitalismus. Es gibt Alternativen zum herrschenden System. München: Pantheon.

Graeber, David. 2013. The Democracy Project. A History, a Crisis, a Movement. New York: Spiegel \& Grau.

Green, Jeffrey Edward. 2013. Rawls and the Forgotten Figure of the Most Advantaged: In Defense of Reasonable Envy toward the Superrich. American Political Science Review 107: 123-138.

Habermas, Jürgen. 2011. Zur Verfassung Europas. Ein Essay. Berlin: Suhrkamp.

Hacker, Jacob S., und Paul Pierson. 2010. Winner-Take-All Politics. How Washington Made the Rich Richer - And Turned Its Back on the Middle Class. New York u. a.: Simon \& Schuster.

Hayek. Friedrich. A. von. 2003. Recht, Gesetz und Freiheit. Eine Neufassung der liberalen Grundsätze der Gerechtigkeit und der politischen Ökonomie. Tübingen: Mohr Siebeck.

Helfrich, Silke, und Heinrich-Böll-Stiftung (Hrsg.). 2012. Commons. Für eine neue Politik jenseits von Markt und Staat. Bielefeld: transcript.

Hessel, Stéphane. 2011. Empört Euch! Berlin: Ullstein.

Hickel, Rudolf. 2012. Zerschlagt die Banken. Zivilisiert die Finanzmärkte. Eine Streitschrift. Berlin: Econ.

Jackson, Ben. 2012. Property-Owning Democracy: A Short History. In Property-Owning Democracy. Rawls and Beyond, Hrsg. Martin O’Neill und Thad Williamson, 33-52. Malden/Oxford: Wiley-Blackwell.

Jaggar, Alison M. (Hrsg.). 2010. Thomas Pogge and His Critics. Cambridge/Malden: Polity Press.

James, Harold. 2009. The Creation and Destruction of Value. The Globalization Cycle. Cambridge/London: Harvard University Press.

Krouse, Richard, und Michael McPherson. 1988. Capitalism, 'Property-Owning Democracy' and the Welfare State. In Democracy and the Welfare State, Hrsg. Amy Gutman, 157-185. Princeton: Princeton University Press.

Lovett, Frank. 2010. A General Theory of Domination and Justice. Oxford: Oxford University Press.

Luhmann, Niklas. 1988. Die Wirtschaft der Gesellschaft. Frankfurt a. M.: Suhrkamp.

Maffettone, Sebastiano. 2010. Rawls. An Introduction. Cambridge/Malden: Polity.

Martens, Helmut. 2010. Neue Wirtschaftsdemokratie. Anknüpfungspunkte im Zeichen der Krise von Ökonomie, Ökologie und Politik. Hamburg: VSA-Verlag.

Meyer, Thomas. 2009. Soziale Demokratie. Eine Einführung. Wiesbaden: VS Verlag für Sozialwissenschaften.

Meyer, Thomas. 2011. Theorie der sozialen Demokratie. 2., durchgesehene und aktualisierte Auflage. Wiesbaden: VS Verlag für Sozialwissenschaften.

Miller, Richard W. 2010. Globalizing Justice. The Ethics of Poverty and Power. Oxford: Oxford University Press. 
Misik, Robert. 2010. Anleitung zur Weltverbesserung. Das machen wir doch mit links. Berlin: Aufbau Verlag.

Münnich, Sascha. 2012. Von Heuschrecken und Bienen. Profit als Legitimationsproblem. In Der Aufstieg der Legitimitätspolitik. Rechtfertigung und Kritik politisch-ökonomischer Ordnungen, Leviathan-Sonderband 27, Hrsg. Anna Geis, Frank Nullmeier und Christopher Daase, 283-301. Baden-Baden: Nomos.

Naphtali, Fritz. 1966. Wirtschaftsdemokratie. Ihr Wesen, Weg und Ziel. Frankfurt a. M.: EVA.

Neckel, Sighard. 2008. Flucht nach vorn. Die Erfolgskultur der Marktgesellschaft. Frankfurt/New York: Campus Verlag.

Neuhäuser, Christian. 2011. Unternehmen als moralische Akteure. Berlin: Suhrkamp.

Norris, Pippa. 2011. Democratic Deficit: Critical Citizens Revisited. Cambridge: Cambridge University Press.

Nozick, Robert. 1974. Anarchy, State, and Utopia. New York: Basic Books.

Nullmeier, Frank. 2000. Politische Theorie des Sozialstaats. Frankfurt/New York: Campus Verlag.

Nullmeier, Frank. 2010. Kritik neoliberaler Menschen- und Weltbilder und Konsequenzen für ein neues Verständnis von, sozialer Gerechtigkeit'. WISO-Diskurs. Expertisen und Dokumentationen zur Wirtschafts- und Sozialpolitik. Bonn: Friedrich-Ebert-Stiftung.

Obinger, Herbert. 2012. Die Finanzkrise und die Zukunft des Wohlfahrtsstaates. Leviathan 40: 441-461.

Oertzen, Peter von, Horst Ehmke und Herbert Ehrenberg (Hrsg.) 1976. Orientierungsrahmen '85. Text und Diskussion. Bonn-Bad Godesberg: Verlag Neue Gesellschaft.

Olsaretti, Serena. (Hrsg.). 2003. Desert and Justice. Oxford: Clarendon Press.

O’Neill, Martin. 2012. Free (and Fair) Markets without Capitalism. Political Values, Principles of Justice, and Property-Owning Democracy. In Property-Owning Democracy. Rawls and Beyond, Hrsg. Martin O’Neill und Thad Williamson, 75-100. Malden/Oxford: Wiley-Blackwell.

O’Neill, Martin, und Thad Williamson (Hrsg.). 2012. Property-Owning Democracy. Rawls and Beyond. Malden/Oxford: Wiley-Blackwell.

Pettit, Philip. 1999. Republicanism. A Theory of Freedom and Government. Oxford: Oxford University Press.

Pogge, Thomas. 2008. World Poverty and Human Rights. Cosmopolitan Responsibilities and Reforms. Second Edition. Cambridge/Malden: Polity Press.

Pogge, Thomas, und Darrel Moellendorf, Darrel (Hrsg.). 2008. Global Justice: Seminal Essays. Global Responsibilities Volume I. St. Paul: Paragon House.

Posner, Richard A. 2009. A Failure of Capitalism. The Crisis of '08 and the Descent into Depression. Cambridge/London: Harvard University Press.

Posner, Richard A. 2010. The Crisis of Capitalist Democracy. Cambridge/London: Harvard University Press.

Rawls, John. 1971. A Theory of Justice. Cambridge/London: Belknap Press of Harvard University Press.

Rawls, John. 1979. Eine Theorie der Gerechtigkeit. Frankfurt a. M.: Suhrkamp.

Rawls, John. 1993. Political Liberalism. New York: Columbia University Press.

Rawls, John. 1998. Politischer Liberalismus. Frankfurt a. M.: Suhrkamp. 
Rawls, John. 2001. Justice as Fairness. A Restatement. Hrsg. von Erin Kelly. Cambridge/ London: Belknap Press of Harvard University Press.

Rawls, John. 2003. Gerechtigkeit als Fairneß. Ein Neuentwurf. Frankfurt a.M.: Suhrkamp Rawls, John. 2007. Lectures on the History of Political Philosophy. Hrsg. von Samuel

Freeman. Cambridge/London: Belknap Press of Harvard University Press.

Roemer, John E. 1994. A Future for Socialism. Cambridge: Harvard University Press.

Ronzoni, Miriam. 2007. Two Concepts of the Basic Structure, and Their Relevance to Global Justice. Global Justice: Theory Practice Rhetoric 1: 68-85.

Ronzoni, Miriam. 2008. What Makes a Basic Structure Just? Res Publica 14: 203-218.

Ronzoni, Miriam. 2012. Life is not a Camping Trip - On the Desirability of Cohenite Socialism. Politics, Philosophy \& Economics 11: 171-185.

Sandel, Michael J. 2012. What Money Can't Buy. The Moral Limits of Markets. New York: Farrar, Straus and Giroux.

Satz, Debra. 2010. Why Some Things Should Not Be for Sale. The Moral Limits of Markets. Oxford: Oxford University Press.

Schäfer, Armin, und Wolfgang Streeck (Hrsg.). 2013: Politics in the Age of Austerity. Cambridge: Polity Press.

Scharpf, Fritz W. 2013. Monetary Union, Fiscal Crisis and the Disabling of Democratic Accountability. In Politics in the Age of Austerity, Hrsg. Armin Schäfer und Wolfgang Streeck, 108-142. Cambridge: Polity Press.

Schaub, Jörg. 2009. Gerechtigkeit als Versöhnung. John Rawls' politischer Liberalismus. Frankfurt/New York: Campus Verlag.

Schemmel, Christian. 2011. Distributive and Relational Equality. Politics, Philosophy \& Economics 11: 123-148.

Schirrmacher, Frank, und Thomas Strobl (Hrsg.). 2010. Die Zukunft des Kapitalismus. Berlin: Suhrkamp.

Schulze, Ingo. 2012. Unsere schönen neuen Kleider. Gegen die marktkonforme Demokratie - für demokratiekonforme Märkte. Berlin: Hanser.

Schweickart, David. 1993. Against Capitalism. Cambridge: Cambridge University Press.

Schweickart, David. 2012. Property-Owning Democracy or Economic Democracy? In Property-Owning Democracy. Rawls and Beyond, Hrsg. Martin O’Neill und Thad Williamson, 200-222. Malden/Oxford: Wiley-Blackwell.

Sen, Amartya. 2009. The Idea of Justice. Cambridge: The Belknap Press of Harvard University Press.

Shiller, Robert J. 2012. Finance and the Good Society. Princeton/Oxford: Princeton University Press.

Skidelsky, Robert. 2009. Keynes: The Return of the Master. London: Penguin Books.

Stiglitz, Joseph. 2012. Der Preis der Ungleichheit. Wie die Spaltung der Gesellschaft unsere Zukunft bedroht. München: Siedler.

Streeck, Wolfgang. 2013. Gekaufte Zeit. Die vertagte Krise des demokratischen Kapitalismus. Berlin: Suhrkamp.

Teubner, Gunther. 2012. Verfassungsfragmente. Gesellschaftlicher Konstitutionalismus in der Globalisierung. Berlin: Suhrkamp.

Tomasi, John. 2012. Free Market Fairness. Princeton/Oxford: Princeton University Press.

Vilmar, Fritz. 1973. Strategien der Demokratisierung. Band I: Theorie der Praxis. Darmstadt/Neuwied: Hermann Luchterhand Verlag. 
Vogl, Joseph. 2010. Das Gespenst des Kapitals. Zürich: Diaphanes.

Walzer, Michael. 1983. Spheres of Justice. A Defense of Pluralism and Equality. New York: Basic Books.

Wingert, Lutz. 2013. Die marktkonforme Demokratie. Alles halb so schlimm? Mittelweg 36 22: 53-67.

Wright, Erik Olin. 2010. Envisioning Real Utopias. London/New York: Verso.

Young, Iris Marion. 1990. Justice and the Politics of Difference. Princeton: Princeton University Press.

\section{Autorenangaben:}

Prof. Dr. Frank Nullmeier,

Universität Bremen, Zentrum für Sozialpolitik (ZeS), Postfach 3304 40,

28334 Bremen,

frank.nullmeier@zes.uni-bremen.de 